\title{
Chronic myeloid leukemia stem cells require cell-autonomous pleiotrophin signaling
}

\author{
Heather A. Himburg, ${ }^{1}$ Martina Roos, ${ }^{1,2}$ Tiancheng Fang, ${ }^{1,3}$ Yurun Zhang, ${ }^{4}$ Christina M. Termini, ${ }^{1}$ Lauren Schlussel, ${ }^{1}$ Mindy Kim, ${ }^{1}$ \\ Amara Pang, ${ }^{1}$ Jenny Kan, ${ }^{1}$ Liman Zhao, ${ }^{1}$ Hyung Suh, ${ }^{1}$ Joshua P. Sasine,,${ }^{1,2}$ Gopal Sapparapu, ${ }^{5}$ Peter M. Bowers, ${ }^{5}$ Gary Schiller, ${ }^{1,2}$ \\ and John P. Chute ${ }^{1,2,6}$ \\ 'Division of Hematology/Oncology, Department of Medicine, UCLA, Los Angeles, California, USA. ${ }^{2} J o n s s o n$ Comprehensive Cancer Center, UCLA, Los Angeles, California, USA. ${ }^{3}$ Department of Molecular \\ and Medical Pharmacology, UCLA, Los Angeles, California, USA. ${ }^{4}$ Molecular Biology Institute, UCLA, Los Angeles, California, USA. ${ }^{5}$ UCLA Clinical and Translational Science Institute, David Geffen School of \\ Medicine, UCLA, Los Angeles, California, USA. ${ }^{6}$ Eli and Edythe Broad Center for Stem Cell Research and Regenerative Medicine, UCLA, Los Angeles, California, USA.
}

Tyrosine kinase inhibitors (TKIs) induce molecular remission in the majority of patients with chronic myelogenous leukemia (CML), but the persistence of CML stem cells hinders cure and necessitates indefinite TKI therapy. We report that CML stem cells upregulate the expression of pleiotrophin (PTN) and require cell-autonomous PTN signaling for CML pathogenesis in BCR/ABL+ mice. Constitutive PTN deletion substantially reduced the numbers of CML stem cells capable of initiating $C M L$ in vivo. Hematopoietic cell-specific deletion of PTN suppressed CML development in BCR/ABL+ mice, suggesting that cell-autonomous PTN signaling was necessary for CML disease evolution. Mechanistically, PTN promoted CML stem cell survival and TKI resistance via induction of Jun and the unfolded protein response. Human CML cells were also dependent on cell-autonomous PTN signaling, and anti-PTN antibody suppressed human CML colony formation and CML repopulation in vivo. Our results suggest that targeted inhibition of PTN has therapeutic potential to eradicate CML stem cells.

\section{Introduction}

The $B C R / A B L$ mutation is the hallmark of chronic myelogenous leukemia (CML), and more than $95 \%$ of patients with this disease demonstrate the $\mathrm{t}(9 ; 22)(\mathrm{q} 34 ; \mathrm{q} 11)$ translocation responsible for generating the BCR/ABL fusion oncoprotein $(1,2)$. The presence of this mutation in all hematopoietic lineages suggested that CML was a stem cell disorder initiated by a mutation in long-term hematopoietic stem cells $(3,4)$. Furthermore, the $B C R / A B L$ mutation was shown to confer leukemic transformation of purified hematopoietic stem cells (HSCs) but failed to transform myeloid progenitors (5). In keeping with the concept of CML as a stem cell disorder, CML stem cells were demonstrated to have the capacity to initiate and reconstitute disease upon serial transplantation $(6,7)$.

CML stem cells possess the capacity to self-renew and differentiate to form aberrant hematopoietic subsets $(6,7)$. Importantly, while tyrosine kinase inhibitor (TKI) treatment induces apoptosis in the bulk of BCR/ABL-expressing tumor cells, quiescent CML stem cells demonstrate resistance to TKI treatment, via preexisting point mutations as well as the acquisition of additional mutations and genomic instability $(3,8-12)$. In addition to cell-autonomous mechanisms of resistance, extrinsic signals from the bone marrow (BM) microenvironment have been described to contribute to CML resistance after TKI therapy (13-23).

Conflict of interest: The authors have declared that no conflict of interest exists. Copyright: (5) 2020, American Society for Clinical Investigation.

Submitted: March 25, 2019; Accepted: September 25, 2019; Published: December 3, 2019. Reference information: J Clin Invest. 2020;130(1):315-328.

https://doi.org/10.1172/JCl129061.
As CML progresses from the chronic phase to blast crisis, leukemic stem cells are no longer restricted to the HSC compartment, and granulocyte-macrophage progenitors can acquire CML stem cell properties via stabilization of nuclear $\beta$-catenin (24). Furthermore, the abnormal CML clone can drive or accentuate niche mechanisms to its own advantage at the expense of normal (NL) hematopoiesis $(7,21)$. However, the contributions of autocrine mechanisms in regulating the $\mathrm{CML}$ pathogenesis are less well understood (25-27). Here, we show that cell-autonomous expression of a heparin-binding growth factor, pleiotrophin (PTN), is necessary for CML pathogenesis and initiation of CML in transplanted mice. PTN is expressed by $\mathrm{BM}$ vascular niche cells to support NL hematopoiesis in healthy mice, whereas CML stem cells upregulate PTN expression and secrete PTN in a cell-autonomous manner to drive CML disease. Antibody-mediated inhibition of PTN suppresses human CML growth in vitro and in vivo, suggesting that PTN is an attractive therapeutic target in human CML.

\section{Results}

$P T N$ is necessary for CML pathogenesis in BCR/ABL-expressing mice. PTN is an HSC growth factor that is secreted by BM stromal cells and endothelial cells (ECs) in healthy mice $(28,29)$. We sought to determine if PTN regulates CML pathogenesis. For this purpose, we utilized the Scl/Tal1-tTA $\times$ TRE-BCR/ABL double-transgenic mice, which allow for inducible $B C R / A B L$ expression in hematopoietic stem/progenitor cells (HSPCs) under the control of doxycycline treatment (2). Scl/Tal1-tTA $\times$ TRE-BCR/ABL mice $(B A$ mice) characteristically develop features of chronic phase CML (leukocytosis, myeloid shift, splenomegaly) within 6 to 8 weeks of discontinuing doxycycline (2). We crossed BA mice with mice 
A

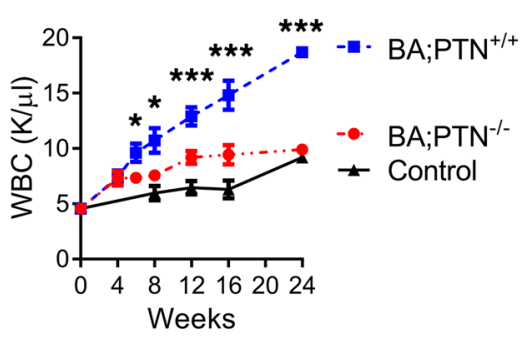

B

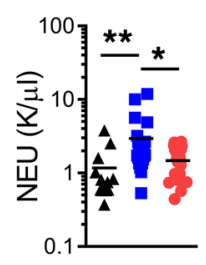

C

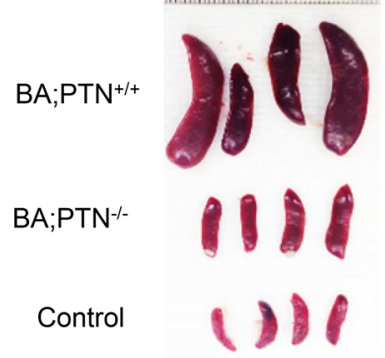

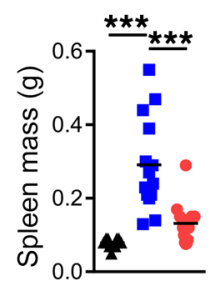

D

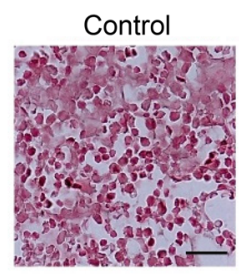

$\mathrm{BA} ; \mathrm{PTN}^{+/+}$

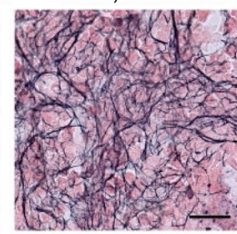

BA; PTN $^{-1-}$

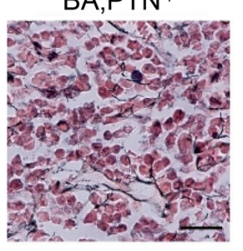

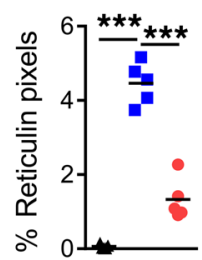

$\mathbf{E}$
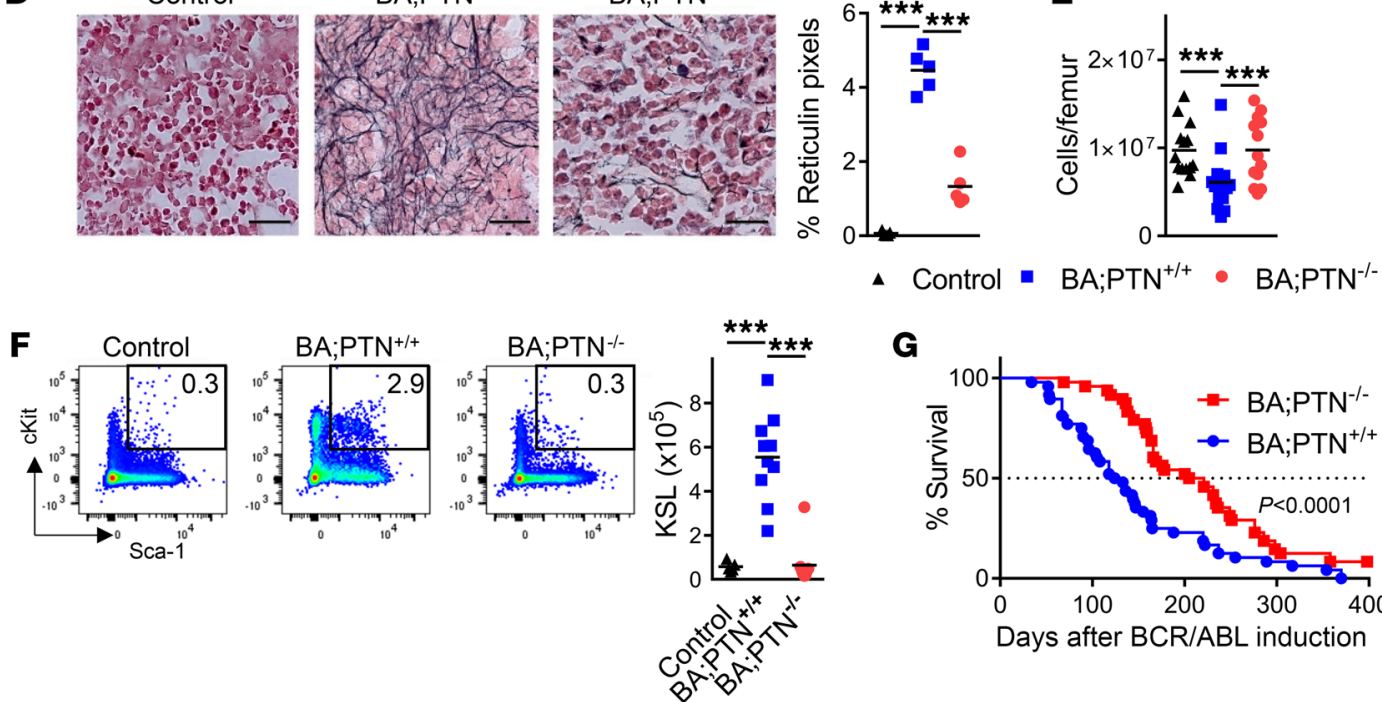

1 Control

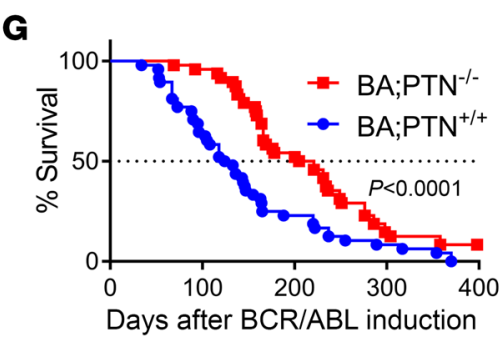

Figure 1. PTN is necessary for CML pathogenesis in BA mice. (A) WBCs over time in adult mice (controls, black), BA;PTN ${ }^{+/+}$mice (blue), and BA;PTN ${ }^{-/-}$mice (red; $n=8-32$ /group). (B) NEUs at 12 weeks after BCR/ABL induction in BA;PTN ${ }^{+/+}$mice, BA;PTN ${ }^{-/-}$mice and controls ( $n=10-23 /$ group). (C) Left: Representative images of spleens at 12 weeks after BCR/ABL induction. Right: Mean spleen mass for each group ( $n=12-14$ mice/group). (D) Left: Representative images of BM reticulin staining (black) in the groups shown at 12 weeks. Right: Percentage reticulin pixels in each group ( $n=5$ mice/group, $\times 63)$. (E) BM cellularity at 12 weeks ( $n=14$ /group). (F) Left: Representative flow cytometric analysis of KSL cells in the spleens at 12 weeks after BCR/ABL induction. Right: Numbers of KSL cells are shown in each group ( $n=5-10 /$ group). (C) Survival of BA;PTN ${ }^{-/-}$mice and BA;PTN $\mathrm{N}^{+/+}$mice $(P<0.0001 ; n=48 /$ group, logrank). $P$ values in were calculated using Tukey's multiple-comparisons test for 2-way ANOVA (A) or Tukey's multiple-comparisons test for 1-way ANOVA (B-F). ${ }^{*} P<0.05,{ }^{* *} P<0.01,{ }^{* *} P<0.001$.

bearing a constitutive deletion of PTN $\left(\mathrm{PTN}^{-/-}\right.$mice) and $\mathrm{PTN}^{+/+}$ control mice to determine the effect of PTN deletion on CML pathogenesis and CML stem cell function in vivo.

PTN-expressing BA mice (BA; $\left.\mathrm{PTN}^{+/+}\right)$demonstrated leukocytosis within 8 weeks following doxycycline withdrawal. At 12 weeks, $\mathrm{BA} ; \mathrm{PTN}^{+/+}$mice displayed substantially increased peripheral blood white blood cell counts (PB WBCs) and neutrophil counts (NEUs) compared with control mice (Figure 1, A and B). Conversely, BA mice bearing PTN deletion (BA; $\mathrm{PTN}^{-} /$mice) displayed NL range $\mathrm{PB}$ WBCs and NEUs that were comparable with control mice (Figure 1, A and B).

$\mathrm{BA} ; \mathrm{PTN}^{+/+}$mice displayed pronounced splenomegaly at 12 weeks following $B C R / A B L$ induction (Figure $1 C$ ), whereas spleens from $\mathrm{BA} ; \mathrm{PTN}^{-/}$mice remained substantially smaller and comparable in size with control mice (Figure 1C). Consistent with this observation, $\mathrm{BA} ; \mathrm{PTN}^{+/+}$mice demonstrated increased splenic mass compared with BA;PTN ${ }^{-/-}$mice and controls (Figure 1C). At 12 weeks after doxycycline withdrawal, BA;PTN ${ }^{+/+}$mice also displayed increased BM fibrosis, a feature of accelerated phase CML (30), whereas $\mathrm{BA} ; \mathrm{PTN}^{-/}$mice displayed only minimal BM fibrosis at this time point (Figure 1D). Commensurate with this finding, $\mathrm{BA} ; \mathrm{PTN}^{+/+}$mice contained significantly decreased $\mathrm{BM}$ cell counts compared with $\mathrm{BA} ; \mathrm{PTN}^{-/-}$mice and controls (Figure 1E).

$\mathrm{BA} ; \mathrm{PTN}^{+/+}$mice displayed increased percentages of myeloid $\left(\mathrm{Mac1}^{+} / \mathrm{Gr}^{+}\right)$cells in the $\mathrm{PB}, \mathrm{BM}$, and spleen compared with control mice, and PTN deletion corrected this myeloid skewing in the $\mathrm{PB}$, with partial correction in the BM and spleen (Supplemental Figure 1, A and B; supplemental material available online with this article; https://doi.org/10.1172/JCI129061DS1). BA;PTN ${ }^{+/+}$mice also demonstrated decreased $\mathrm{B}$ lymphocytes in the BM compared with control mice, whereas $\mathrm{BA} ; \mathrm{PTN}^{-/-}$mice displayed increased B cell percentages compared with $\mathrm{BA} ; \mathrm{PTN}^{+/+}$mice (Supplemental Figure 1B). CML stem cells are enriched within the ckit ${ }^{+} \mathrm{sca}-1^{+}$ lin $^{-}$(KSL) population and the $\mathrm{CD}^{150^{+}} \mathrm{CD}^{-} 8^{-} \mathrm{KSL}$ population (23). BA; $\mathrm{PTN}^{+/+}$mice demonstrated increased percentages and numbers of KSL cells and $\mathrm{CD} 15 \mathrm{O}^{+} \mathrm{CD} 48^{-} \mathrm{KSL}$ cells in the spleen compared with control mice (Figure $1 \mathrm{~F}$ and Supplemental Figure 2, $\mathrm{A}$ and $\mathrm{B}$ ). In contrast, $\mathrm{BA} ; \mathrm{PTN}^{--}$mice showed no increase in KSL cells or $\mathrm{CD}^{150^{+}} \mathrm{CD} 48^{-} \mathrm{KSL}$ cells compared with control mice 
A

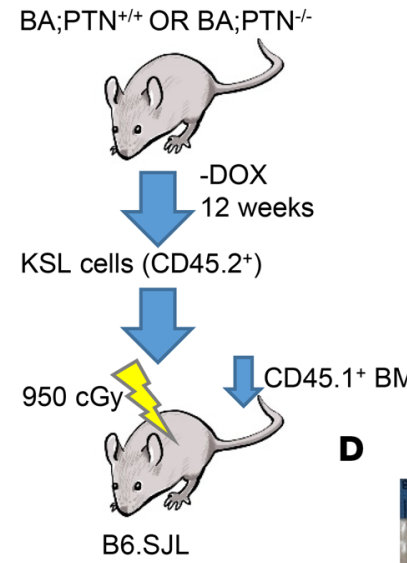

B

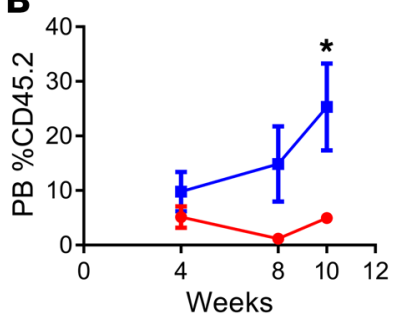

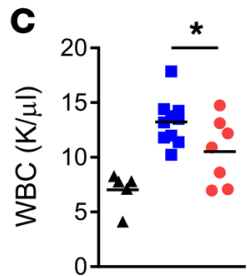

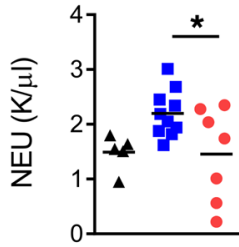

A Control - BA;PTN ${ }^{+/+}$BA;PTN ${ }^{-1-}$
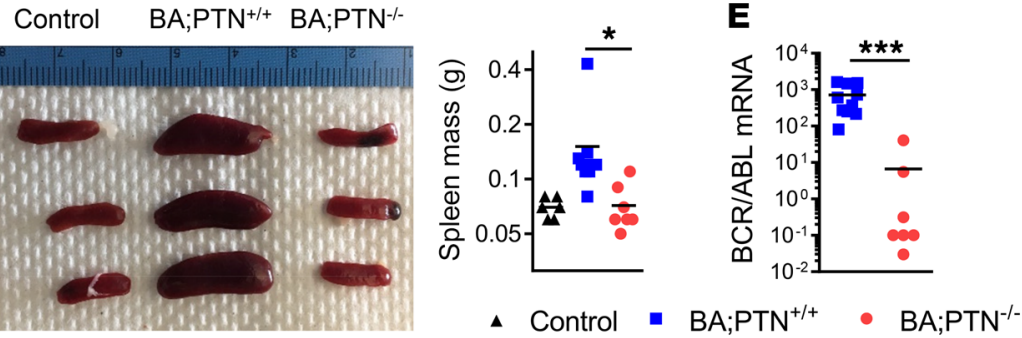

$\mathbf{F}$
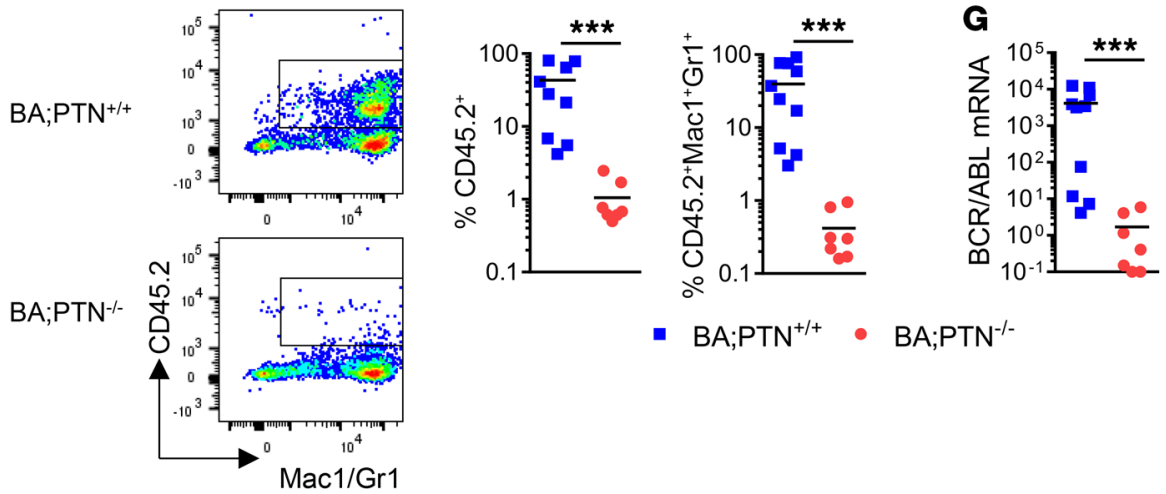

- BA;PTN ${ }^{+/+}$• BA;PTN ${ }^{-/-}$

Figure 2. PTN deletion impairs CML stem cell repopulating capacity. (A) Experimental design. (B) Donor CD45.2 $2^{+}$cell engraftment in PB of recipient mice transplanted with splenic KSL cells from BA;PTN ${ }^{+/+}$mice or BA;PTN ${ }^{-1-}$ mice ( $n=7-10$ mice/time point). (C) PB WBCs and neutrophils in the recipient mice at 10 weeks after transplant, along with controls ( $n=5-10 /$ group). (D) Left: Representative images of spleens from recipient mice transplanted with KSL cells from BA;PTN ${ }^{+/+}$or BA;PTN ${ }^{-/-}$mice at 10 weeks after transplant, along with controls. Right: Mean spleen masses for each group ( $\left.n=6-10 / g r o u p\right)$. (E) $B C R /$ $A B L$ transcript levels in the spleens of recipient mice ( $n=7-9$ per group). (F) Left: Representative flow cytometric analysis of donor myeloid (Mac1/Gr1 $\left.{ }^{+}\right)$ cell engraftment in the BM of recipient mice. Right: Percentage BM total CD45.2+ cell and donor myeloid cell engraftment in each group ( $n=7-10 /$ group). (C) $B C R / A B L$ transcript levels in the BM of recipient mice ( $n=7-9$ per group). $P$ values were calculated using Sidak's multiple-comparisons test for 2-way ANOVA (B) or 2-tailed Student's $t$ test $(\mathbf{C}-\mathbf{C}) .{ }^{*} P<0.05,{ }^{* * *} P<0.001$.

(Figure $1 \mathrm{~F}$ and Supplemental Figure 2, $\mathrm{A}$ and $\mathrm{B}) . \mathrm{BA} ; \mathrm{PTN}^{+/+}$mice also displayed increased percentages of common myeloid progenitors, megakaryocyte-erythroid progenitors, and granulocytemonocyte progenitors in the spleen, consistent with CML evolution. BA;PTN ${ }^{-/}$demonstrated no significant increases in these populations (Supplemental Figure 2, A and C).

The majority of BA mice die from CML morbidities within several months after doxycycline withdrawal (2). In our studies, the median survival of $\mathrm{BA} ; \mathrm{PTN}^{+/+}$mice was 129 days (Figure 1G). Conversely, the median survival of $\mathrm{BA} ; \mathrm{PTN}^{-/-}$mice was 213 days. The overall survival of $\mathrm{BA} ; \mathrm{PTN}^{-/-}$mice was highly significantly increased compared with that of $\mathrm{BA} ; \mathrm{PTN}^{+/+}$mice $(P<0.0001$, log-rank test). BA; $\mathrm{PTN}^{-/-}$mice developed leukocytosis and splenomegaly comparable to $\mathrm{BA} ; \mathrm{PTN}^{+/+}$mice, but these morbidities occurred at later time points.
PTN deletion reduces CML stem cells. Because PTN deletion inhibited CML progression in BA mice, we sought to determine if PTN deletion would also affect CML stem cells capable of reconstituting disease in secondary transplanted mice. In order to test this hypothesis, we isolated KSL cells from the spleens of $\mathrm{BA} ; \mathrm{PTN}^{+/+}$mice and $\mathrm{BA} ; \mathrm{PTN}^{-/}$mice at 12 weeks following $B C R / A B L$ induction and competitively transplanted equal numbers of KSL cells into irradiated, congenic recipient mice, as previously described (Figure 2A and ref. 21). At 10 weeks following transplantation, recipients of $1 \times 10^{4} \mathrm{KSL}$ cells from $\mathrm{BA} ; \mathrm{PTN}^{+/+}$mice demonstrated more than $20 \%$ donor $\mathrm{CD} 45.2^{+}$cell engraftment in the $\mathrm{PB}$, whereas recipients of the identical dose of KSL cells from BA;PTN ${ }^{-/}$mice displayed less than $5 \%$ donor cell engraftment (Figure 2B). Recipients of KSL cells from $\mathrm{BA} ; \mathrm{PTN}^{+/+}$mice also displayed significantly increased PB WBCs, NEUs, and splenomegaly at 10 weeks after transplant, consistent 
A

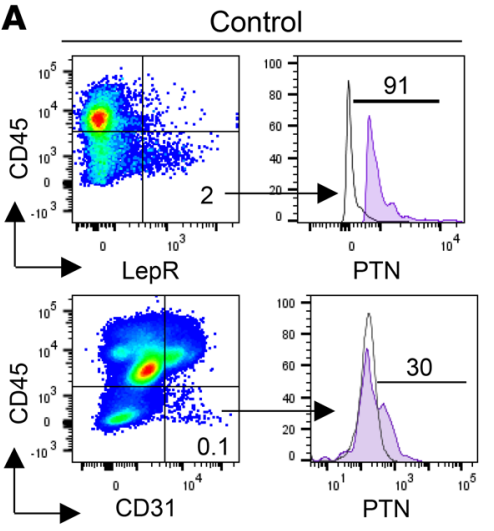

C

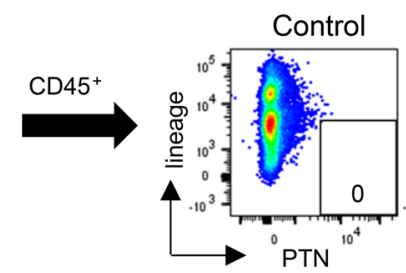

D

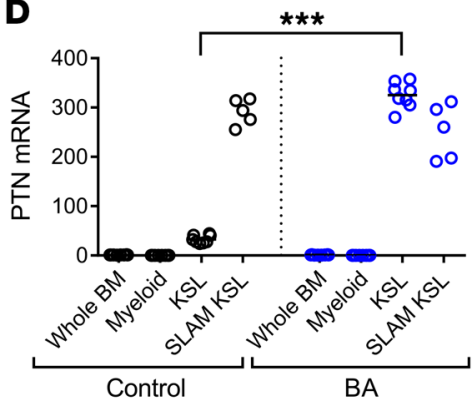

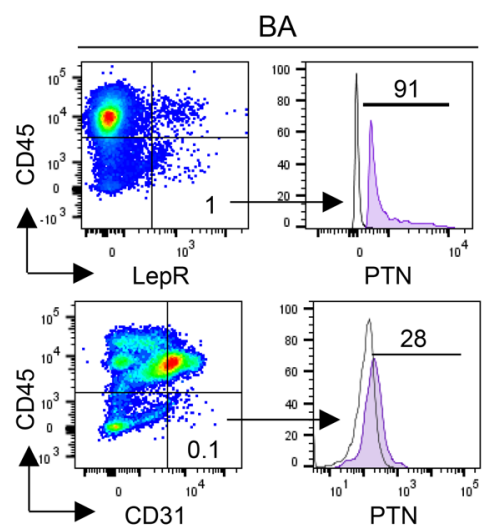

BA

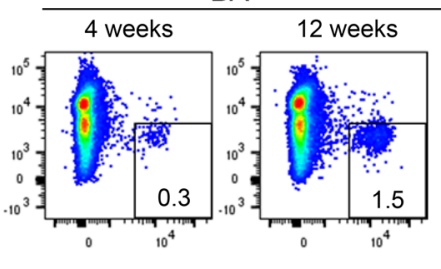

E

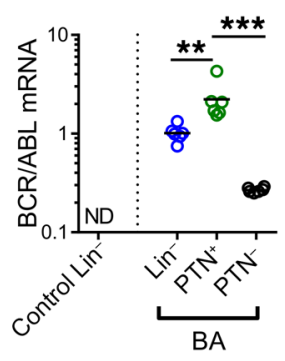

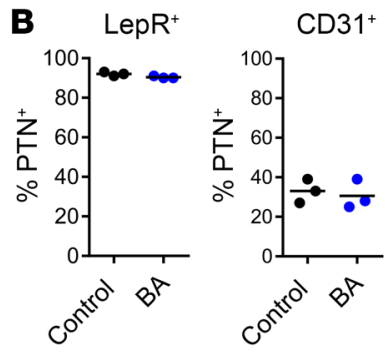

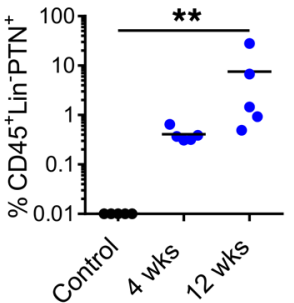

Figure 3. CML stem cells upregulate PTN expression. (A) Representative flow cytometric analysis of PTN expression within splenic CD45-LepR ${ }^{+}$ stromal cells (top) and CD45-CD31+ ECs (bottom) in control mice and in BA mice at 12 weeks after $B C R / A B L$ induction. (B) Percentage PTN ${ }^{+}$cells within the CD45-LepR ${ }^{+}$cells and in CD45-CD31+ ECs in each group. (C) Left: Flow cytometric analysis of PTN expression on splenic CD45 cells from healthy controls and BA mice at 4 and 12 weeks after BCR/ABL induction. Right: Percentage CD45+lin-PTN ${ }^{+}$cells in the different groups ( $n=5$ mice/group).

(D) PTN gene expression in BM cell populations (whole BM, Mac1 $1^{+} / \mathrm{Gr} 1^{+}$myeloid cells, KSL cells and CD150 CD48/41-KSL, SLAM KSL cells) from healthy controls and BA mice at 12 weeks after $B C R / A B L$ induction ( $n=5-8$ mice/group). (E) $B C R / A B L$ transcript levels in the splenic cell populations shown from healthy control mice and BA mice at 12 weeks after $B C R / A B L$ induction. ND, not detected ( $n=6 /$ group). $P$ values in C-E were calculated using Tukey's multiple-comparisons test for 1-way ANOVA. ${ }^{* *} P<0.01,{ }^{* *} P<0.001$.

with the initiation of CML (Figure 2, C and D). In contrast, mice transplanted with equal doses of KSL cells from BA;PTN ${ }^{-/}$mice displayed significantly decreased WBCs and neutrophils, smaller spleen sizes, and spleen mass compared with BA;PTN ${ }^{+/+}$mice (Figure 2, C and D). Furthermore, mice transplanted with KSL cells from BA;PTN ${ }^{+/+}$ donor mice demonstrated $B C R / A B L$ transcript levels in the spleens that were more than 100-fold higher than $B C R / A B L$ transcript levels in recipients of KSL cells from $\mathrm{BA} ; \mathrm{PTN}^{-/}$mice (Figure 2E). These data suggested that deletion of PTN substantially decreased CML stem cells capable of reconstituting CML in transplanted mice.

Donor CD $45.2^{+}$cell engraftment and myeloid skewing were significantly increased in the BM and spleens of mice transplanted with KSL cells from $\mathrm{BA} ; \mathrm{PTN}^{+/+}$mice compared with recipients of KSL cells from $\mathrm{BA} ; \mathrm{PTN}^{-/}$mice (Figure $2 \mathrm{~F}$ and Supplemental Figure $3 \mathrm{~A})$. Donor CD $45.2^{+}$cell engraftment within the BM KSL stem/progenitor cell population was also significantly increased in recipients transplanted with KSL cells from $\mathrm{BA} ; \mathrm{PTN}^{+/+}$cells compared with recipients of KSL cells from BA; $\mathrm{PTN}^{-/-}$mice (Supplemental Figure 3B). Finally, $B C R / A B L$ transcript levels were more than 1,000fold increased in the BM of mice transplanted with KSL cells from $\mathrm{BA} ; \mathrm{PTN}^{+/+}$mice compared with $B C R / A B L$ levels in mice transplanted with KSL cells from $\mathrm{BA} ; \mathrm{PTN}^{-/}$mice (Figure $2 \mathrm{G}$ ).

CML stem cells upregulate PTN expression in BA mice. In NL hematopoiesis, BM stromal cells and ECs secrete PTN in the HSC niche and PTN regulates HSC maintenance and regeneration (29). We sought to determine the cellular source of PTN that supported CML pathogenesis in BA mice. Utilizing PTN-GFP mice crossed with healthy control mice and BA mice, we found that the expression levels of PTN in splenic stromal cells and ECs were not different between control mice and BA mice at 12 weeks following $B C R / A B L$ induction (Figure 3, A and B). Approximately $90 \%$ of CD45-LepR ${ }^{+}$stromal cells expressed PTN in healthy control mice 
A

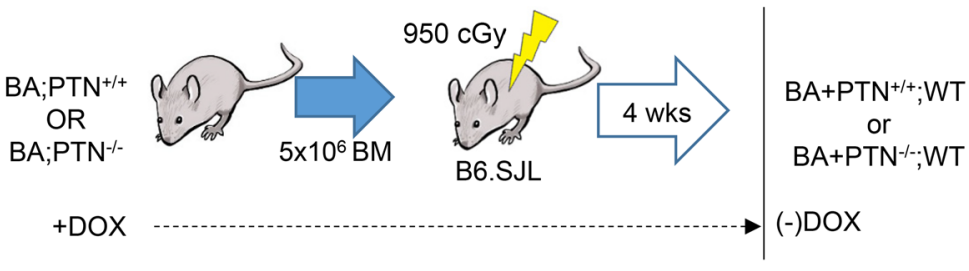

B

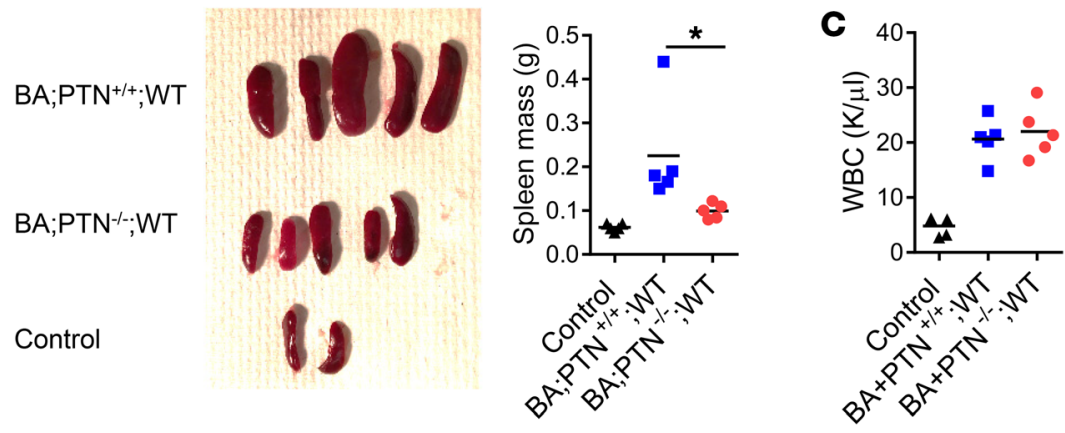

D

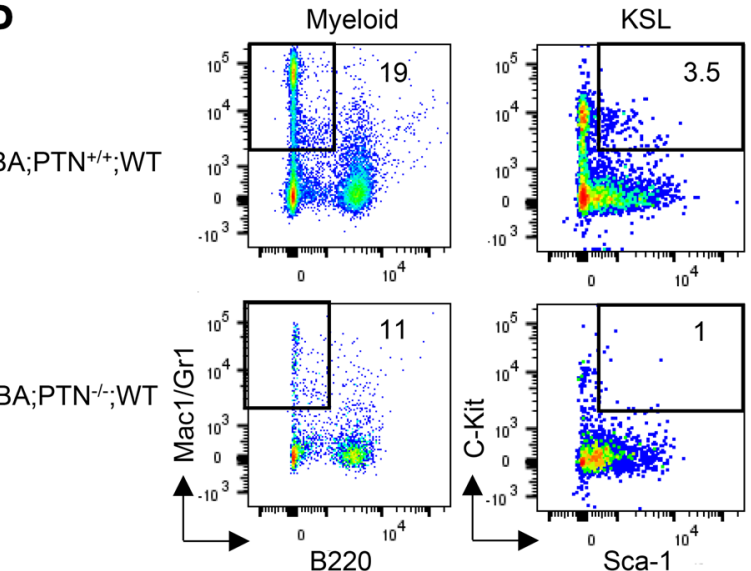

E

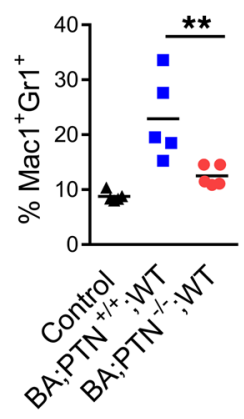

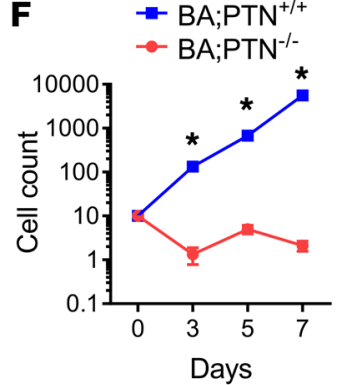

Figure 4. PTN regulates $C M L$ pathogenesis in a hematopoietic cell-autonomous manner. (A) Generation of chimeric mice with PTN deletion restricted to the hematopoietic compartment. (B) Left: Spleens from BA;PTN ${ }^{++} ;$WT mice and BA;PTN ${ }^{-1-}$;WT mice at 16 weeks after $B C R / A B L$ induction, along with healthy controls (Control). Right: Spleen masses are shown ( $n=5$ /group). (C) PB WBCs at 16 weeks in each group $(n=5)$. (D) Flow cytometric analysis of $\mathrm{Mac} 1^{+} / G r 1^{+}$myeloid cells and KSL cells in the spleens of the mice shown at 16 weeks after $B C R / A B L$ induction. (E) Mean percentages of myeloid cells and KSL cells in the spleens of $\mathrm{BA} ; \mathrm{PTN}^{+/+} ; \mathrm{WT}$ mice and $\mathrm{BA} ; \mathrm{PTN}^{-/-} ; \mathrm{WT}$ mice at 16 weeks after $B C R / A B L$ induction, compared with B6.SJL mouse controls ( $n=5 /$ group). (F) Total cell counts over time in liquid culture of $10 \mathrm{KSL}$ cells from BA;PTN ${ }^{+/+}$mice and BA;PTN ${ }^{-1-}$ mice $(n=6-9 /$ group). $P$ values were calculated using 2-tailed Student's $t$ test (B and $\mathbf{E}$ ) or the Holm-Sidak multiple $t$ test $(\mathbf{F}) .{ }^{*} P<0.05,{ }^{* *} P<0.01$.

and in BA mice. Similarly, $28 \%$ of $\mathrm{CD} 45^{-} \mathrm{CD} 31^{+}$ECs expressed PTN in BA mice and $30 \%$ of ECs in control mice expressed PTN (Figure 3, A and B). Of note, in C57BL/6 control mice, CD45 ${ }^{+}$lin hematopoietic cells did not express PTN (Figure 3C). In BA mice, however, $\mathrm{CD}_{4} 5^{+}$lin $^{-}$hematopoietic cells significantly upregulated PTN levels at 4 weeks and 12 weeks following $B C R / A B L$ induction (Figure 3C). Consistent with these findings, PTN gene expression was markedly increased in KSL stem/progenitor cells in BA mice at 12 weeks after $B C R / A B L$ induction compared with KSL cells in control mice (Figure $3 \mathrm{D}$ ). Of note, $\mathrm{CD} 150^{+} \mathrm{CD} 48^{-} \mathrm{KSL}$ cells from both BA mice and C57BL/6 mice express the PTN transcript. Importantly, $\mathrm{PTN}^{+} \mathrm{CD} 45^{+}$lin $^{-}$cells expressed high levels of $B C R / A B L$ in BA mice, whereas $B C R / A B L$ expression was nearly undetectable in $\mathrm{PTN}^{-} \mathrm{CD} 45^{+}$lin $^{-}$cells (Figure $3 \mathrm{E}$ ). Taken together, these results suggest that CML stem cells upregulate PTN expression early during disease development.
In the spleens of healthy C57BL/6 mice, we observed that PTN expression was confined to vascular structures within the red pulp and colocalized with VE-cad ${ }^{+}$ECs and LepR ${ }^{+}$stromal cells (Supplemental Figure 4A). However, in BA mice, we observed a population of bright $\mathrm{PTN}^{+}$cells within the spleen that was distinct from VE-cad ${ }^{+}$blood vessels (Supplemental Figure $4 \mathrm{~B})$. These PTN-expressing cells within the spleen of BA mice colocalized with LepR expression (Supplemental Figure 4B). Using flow cytometric analysis, we further defined this population of $\mathrm{PTN}^{+} \mathrm{LepR}^{+}$cells within the $\mathrm{CD} 45^{+}$hematopoietic population in the spleens of BA mice (Supplemental Figure 4C). Quantitative reverse transcription PCR analysis confirmed that $\mathrm{PTN}^{+}$cells within the spleens of BA mice expressed PTN transcript and also expressed high levels of LepR (Supplemental Figure 5). Taken together, this analysis suggested that a subset of $\mathrm{CD} 45^{+}$hematopoietic cells in BA mice expressed both PTN and 


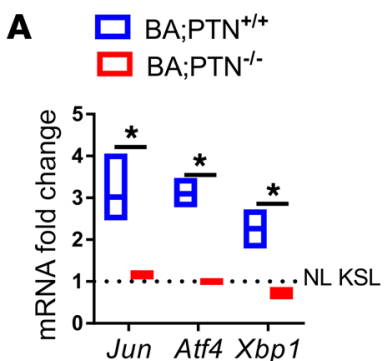

D
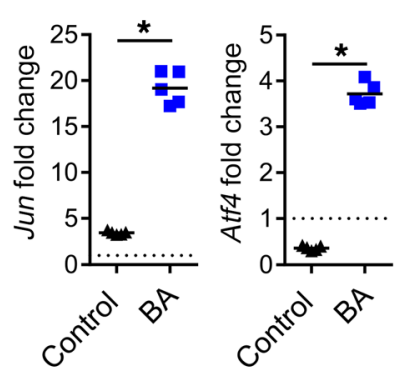
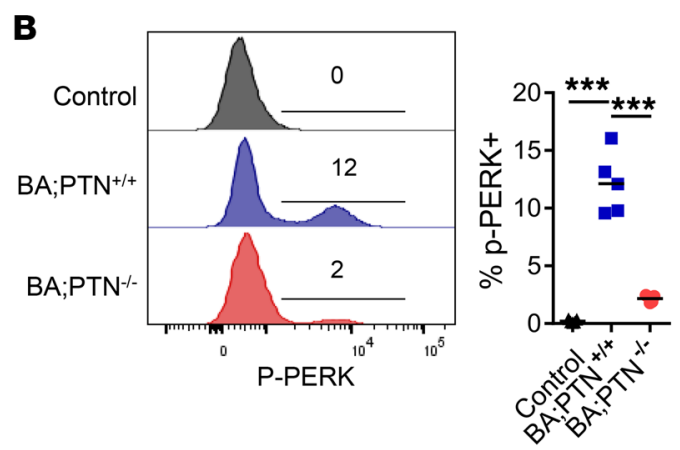

C

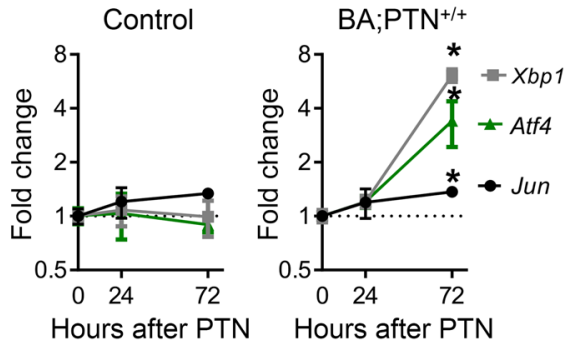

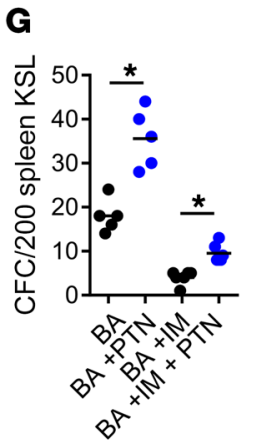

H

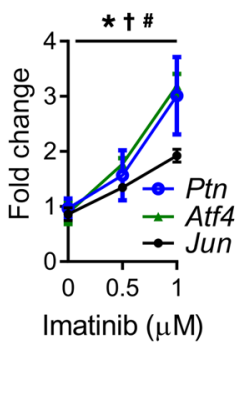

E

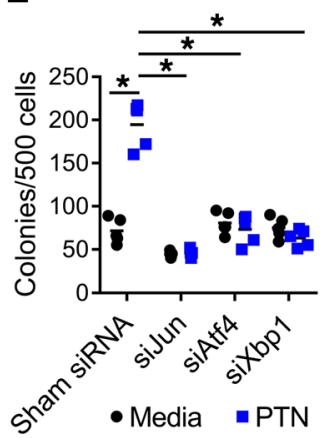

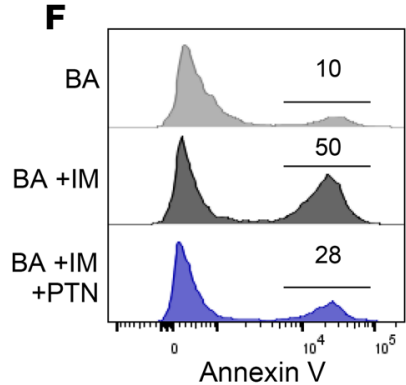

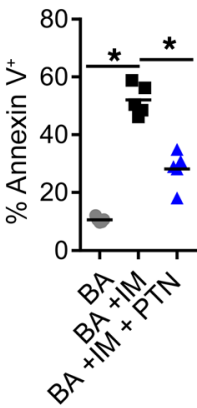

I

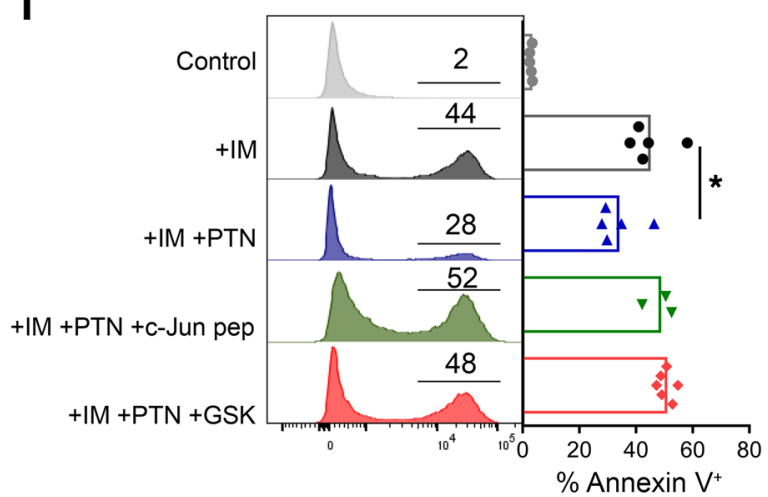

Figure 5. PTN promotes CML stem cell survival via induction of c-Jun and the UPR. (A) Jun, Atf4, and Xbp1 expression in splenic KSL cells at 12 weeks after $B C R / A B L$ induction (dotted line = expression in KSL cells from NL C57BL/6 mice. (B) Left: Histograms of p-PERK in BM KSL cells from BA;PTN ${ }^{+/+}$mice and $\mathrm{BA} ; \mathrm{PTN}^{-1-}$ mice at 12 weeks after BCR/ABL induction, compared with C57BL/6 controls. Right: Percentage p-PERK ${ }^{+}$cells within the KSL population (n = 5/group). (C) Jun, Atf4, and Xbp1 expression in KSL cells from control C57BL/6 mice and from BA; $\mathrm{PTN}^{+/+}$mice treated with $100 \mathrm{ng} / \mathrm{mL} \mathrm{PTN}$ in vitro ( $n=4 /$ group). (D) Fold change in Jun, Atf4, and Xbp1 mRNA expression at 2 hours following treatment with $10 \mu \mathrm{M}$ anisomycin in KSL cells isolated from control C57BL/6 mice and BA mice (dotted line = baseline expression of each gene prior to anisomycin treatment). (E) Leukemic colonies from K562 cells following 72-hour incubation with Jun, Atf4, or Xbp1 siRNA or sham siRNA \pm PTN ( $n=5 /$ group). (F) Annexin V analysis of splenic KSL cells from BA mice treated for 24 hours with IM with or without PTN ( $n=5$ /group). (C) Leukemic colonies (CFCs) from splenic KSL cells from BA mice treated \pm PTN with or without IM ( $n$ $=5 /$ group, 2-tailed Student's $t$ test). (H) Jun, Atf4, and PTN expression in splenic KSL cells from BA mice in response to IM ( $n=5-6 /$ group; $P$ tn: ${ }^{*} P<0.05$; Atf4: ${ }^{\dagger} P<0.05$; Jun: ${ }^{\#} P<0.05$ ). (I) Annexin $V$ analysis of $K 562$ cells treated with IM alone or IM with PTN, with and without the c-Jun peptide inhibitor or GSK2606414 (GSK, $n=3-6 /$ group). $P$ values were calculated using 2-tailed $t$ test (A, D, and $\mathbf{G}$ ), Dunnett's multiple-comparisons test for 2-way ANOVA (C and $\mathbf{E}$ ), or Dunnett's multiple-comparisons test for 1 -way ANOVA (B, F, H, and $\mathbf{I}$ ). ${ }^{*} P<0.05,{ }^{* *} P<0.001$.

LepR. Landberg et al. recently identified LepR as a marker for primitive human $\mathrm{CD} 34^{+} \mathrm{CD} 38^{-} \mathrm{CML}$ cells, which were resistant to imatinib treatment (31).

PTN regulates CML pathogenesis in a hematopoietic cellautonomous manner. In order to determine the cellular source of PTN that governed CML progression in BA mice, we generated chimeric mice using $\mathrm{BM}$ transplantation. We transplanted $5 \times 10^{6}$ $\mathrm{BM}$ cells from $\mathrm{BA} ; \mathrm{PTN}^{+/+}$mice $\left(\mathrm{CD} 45.2^{+}\right)$and $\mathrm{BA} ; \mathrm{PTN}^{-/-}$mice into lethally irradiated B6.SJL $\left(\mathrm{CD} 45.1^{+}\right) \mathrm{WT}$ congenic mice to create chimeric $\mathrm{BA} ; \mathrm{PTN}^{+/} ; \mathrm{WT}$ mice and $\mathrm{BA} ; \mathrm{PTN}^{-/} ; \mathrm{WT}$ mice, in which PTN deletion was restricted to the hematopoietic cell compartment (Figure 4A). After verification of greater than $90 \%$ donor chimerism in all mice at 4 weeks after transplantation, doxycycline was withdrawn from recipient mice to induce $B C R / A B L$ expression in hematopoietic cells only (Supplemental Figure 6A). At 16 weeks following $B C R / A B L$ induction, BA; $\mathrm{PTN}^{++} ; \mathrm{WT}$ mice displayed marked splenomegaly and increased splenic mass (Figure $4 \mathrm{~B})$. Conversely, $\mathrm{BA} ; \mathrm{PTN}^{-/}$;WT mice demonstrated decreased 
A
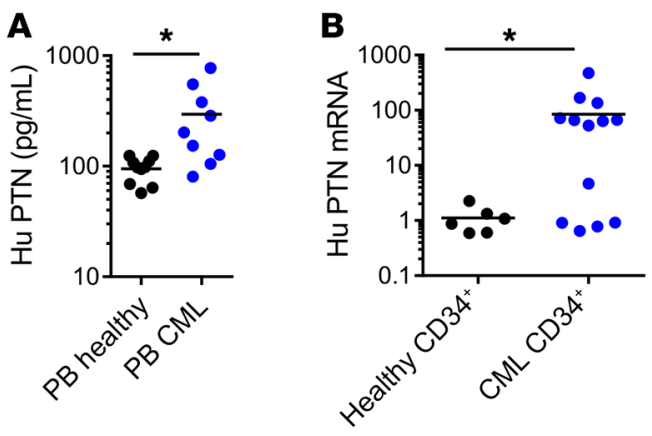

E
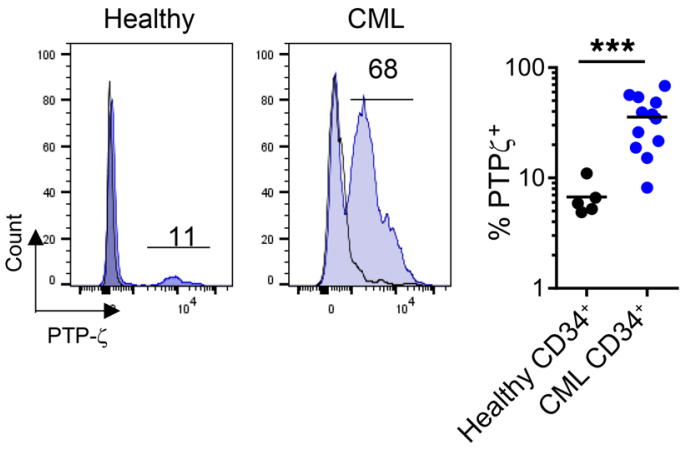
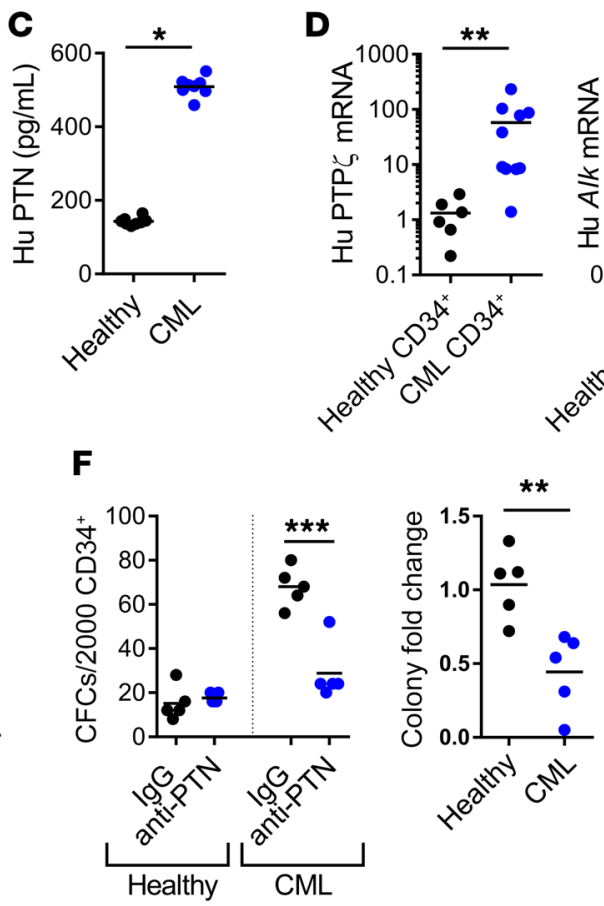

G

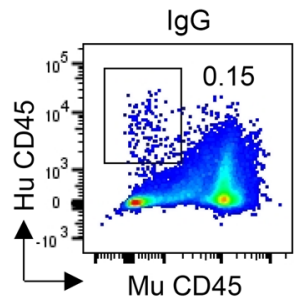

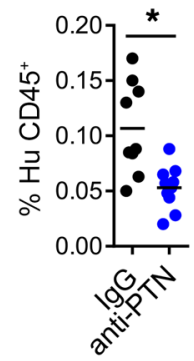

H

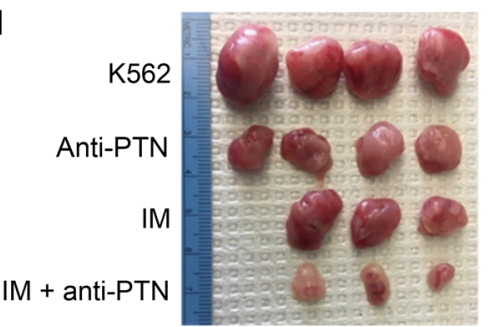

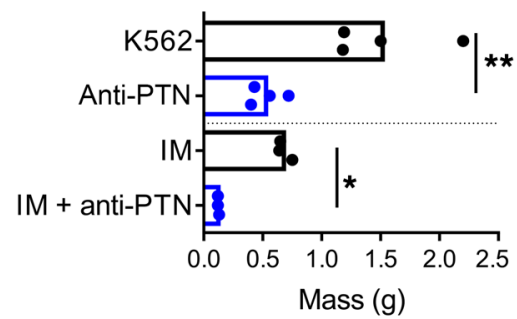

Figure 6. Anti-PTN antibody suppresses human CML stem cell function. (A) Human PTN levels in PB of healthy adults and patients with $C M L$ ( $n=$ 8-10/group). (B) Human PTN gene expression in BM CD34+ cells from healthy adults $(n=6)$ and CD34+ cells from patients with CML ( $n=13$ ). (C) ELISA of conditioned media from 72-hour cultures of BM cells from healthy adults and PB mononuclear cells from patients with CML ( $n=8$ wells/group from 3 samples/group). (D) Expression of PTP- $\zeta$ and ALK mRNA in BM CD34+ cells from healthy adults $(n=6)$ and CD34 cells from patients with CML $(n=10)$. (E) Left: Representative histograms of PTP- $\zeta$ cell surface expression on CD34+ cells from healthy adults and patients with CML. Right: Percentage PTP- $\zeta$ cells ( $n=5$ healthy and $n=12 \mathrm{CML}$ ). (F) Left: CFCs from CD34+ cells treated in vitro with or without $50 \mu \mathrm{g} / \mathrm{mL}$ anti-PTN antibody (healthy donors, CML donors). Right: Fold differences in CFCs from CD34+ cell samples from healthy adults and patients with CML treated with anti-PTN or IgC ( $n=5$ donors/ group). Fold changes are relative to the IgG control. (C) Left: Flow cytometric analysis of human CD45 CML cells in NSG mice at 16 weeks after transplantation. Right: Mean human CD45+ cell engraftment at 16 weeks ( $n=9-10$ mice/group). (H) Left: Tumors from NSG mice at 2 weeks after injection of K562 cells cultured with or without IM for 72 hours. Mice were treated systemically with anti-PTN or IgC for 2 weeks. Right: Tumor masses are shown ( $n=3-4$ mice/group). $P$ values were calculated using Mann-Whitney $U$ test (A-E) or 2-tailed Student's $t$ test $(\mathbf{F}-\mathbf{H}) .{ }^{*} P<0.05,{ }^{* *} P<0.01,{ }^{* * *} P<0.001$.

splenomegaly and spleen mass compared with the $\mathrm{BA} ; \mathrm{PTN}^{+/+} ; \mathrm{WT}$ mice (Figure 4B). PB WBCs were increased in both BA;PTN ${ }^{+/} ; \mathrm{WT}$ mice and $\mathrm{BA} ; \mathrm{PTN}^{-/} ; \mathrm{WT}$ mice compared with control mice (Figure $4 \mathrm{C}$ ), but $\mathrm{BA} ; \mathrm{PTN}^{++} ; \mathrm{WT}$ mice displayed increased myeloid skewing in the $\mathrm{BM}$ and spleens compared with $\mathrm{BA} ; \mathrm{PTN}^{-/} ; \mathrm{WT}$ mice (Figure 4, D and E and Supplemental Figure 6B). BA;PTN ${ }^{+/+}$; WT mice also displayed increased percentages of KSL cells, enriched for CML stem cells (21) in the spleen at 16 weeks following $B C R / A B L$ induction (Figure 4, D and $\mathrm{E}$ ). In contrast, $\mathrm{BA} ; \mathrm{PTN} \mathrm{N}^{-/}$; WT mice demonstrated no increase in KSL cell frequency. Taken 
together, these results suggested that PTN regulates CML pathogenesis primarily in a hematopoietic cell-autonomous manner, with less contribution from niche-derived PTN to CML pathogenesis. Consistent with these results, KSL cells from $\mathrm{BA} ; \mathrm{PTN}^{-/-}$mice displayed no growth in 7-day cultures with thrombopoietin (TPO) and stem cell factor (SCF), whereas KSL cells from $\mathrm{BA} ; \mathrm{PTN}^{+/+}$mice expanded more than 1,000-fold in 7 days (Figure $4 \mathrm{~F}$ ). These data suggest that cell-autonomous PTN signaling is necessary for CML stem cell growth and CML pathogenesis in vivo.

PTN promotes CML pathogenesis via induction of c-Jun and the unfolded protein response. In order to elucidate the mechanisms through which PTN promotes CML pathogenesis, we measured the expression of several leukemia-related genes within splenic KSL cells from $\mathrm{BA} ; \mathrm{PTN}^{-/-}$mice and $\mathrm{BA} ; \mathrm{PTN}^{+/+}$mice at 12 weeks postinduction of $B C R / A B L$ expression, using the Qiagen Leukemia RT2 Profiler (Supplemental Figure 7A). We detected increased expression of Jun, which encodes for transcription factor c-Jun in KSL cells from $\mathrm{BA} ; \mathrm{PTN}^{+/+}$mice, whereas KSL cells from $\mathrm{BA} ; \mathrm{PTN}^{-/}$ mice expressed significantly lower levels of Jun (Supplemental Figure 7A). Quantitative reverse transcription PCR analysis confirmed increased expression of Jun in KSL cells from BA;PTN ${ }^{+/+}$ mice, whereas Jun expression in KSL cells from BA;PTN ${ }^{-/}$mice was not increased compared with KSL cells from healthy controls (Figure 5A). Overexpression of Jun has been implicated in promoting the survival of acute myeloid leukemia (AML) cells $(32,33)$. It has been suggested that c-Jun promotes AML cell survival, in part, by inducing the transcription of key effectors of the unfolded protein response (UPR), including Xbp1 and Atf4 (34). UPR activation encompasses an ordered set of cellular responses required to restore endoplasmic reticulum (ER) homeostasis, including cessation of protein synthesis, regulation of apoptosis, and upregulation of corrective transcriptional programs (34-36). In AML, c-Jun-induced expression of the UPR effectors, Xbp1 and Atf4, produces antiapoptotic effects (34). We found that KSL cells in $\mathrm{BA} ; \mathrm{PTN}^{+/+}$mice expressed increased levels of Jun and Xbp1 and Atf4, whereas PTN-deficient KSL cells showed no increase in Jun, Xbp1, or Atf4 expression in KSL cells (Figure 5A). KSL cells from $\mathrm{BA} ; \mathrm{PTN}^{+/+}$mice also displayed markedly increased levels of phospho-protein kinase R-like ER kinase (p-PERK), which activates Atf4 in the setting of ER stress, compared with KSL cells from $\mathrm{BA} ; \mathrm{PTN}^{-/-}$mice $(34,37$, 38) (Figure 5B). Treatment of KSL cells from BA mice with $100 \mathrm{ng} /$ $\mathrm{mL}$ PTN for 72 hours also caused a significant increase in expression of Jun, Xbp1, and Atf4 in KSL cells, whereas PTN treatment of KSL cells from C57BL/6 mice caused no significant changes in Jun, $X b p 1$, or Atf4 expression (Figure 5C). In order to determine if Jun induction differentially increased Atf 4 and $X b p 1$ expression in KSL cells from BA mice compared with KSL cells from C57BL/6 mice, we treated both populations with $10 \mu \mathrm{M}$ anisomycin, a potent and specific inducer of Jun expression (39) and measured Atf4 and Xbp1 expression. Jun expression increased greater than 4-fold in KSL cells from BA mice in response to anisomycin compared with control KSL cells. Furthermore, Xbp1 and Atf4 expression increased significantly in KSL cells from BA mice in response to anisomycin, whereas expression of $X b p 1$ and Atf 4 did not increase in control KSL cells (Figure 5D). Treatment of KSL cells from BA mice for 72 hours with Jun siRNA also significantly decreased Xbp1 and Atf4 expression compared with siRNA control-treated KSL cells, confirming the molecular linkage between Jun and Xbp1 and Atf4 expression in CML stem cells (Supplemental Figure 7B). Of note, c-Jun is part of the AP1 transcription factor complex and 2 AP1 binding sites have been identified in the $5^{\prime}$ promoter region of the PTN gene (40). Expression of the BCR/ABL fusion protein increases c-Jun activation and c-Jun activation is required for BCR/ABL-mediated cellular transformation (41). We found that anisomycin-mediated induction of Jun expression significantly increased PTN expression in KSL cells from BA mice (Supplemental Figure 7C). These results suggest that c-Jun may directly promote increased transcription of PTN in CML stem cells.

In order to determine if c-Jun or the UPR proteins, Xbp1 or Atf4, were required for PTN-mediated promotion of CML growth, we treated human K562 CML cells (42) with siRNAs targeting Jun, Atf4, and Xbp1, and compared leukemic colony cell formation in response to $100 \mathrm{ng} / \mathrm{mL} \mathrm{PTN}$ treatment (Figure 5E). PTN treatment significantly increased CML colony formation at 72 hours, whereas silencing of Jun, Xbp1, or Atf 4 abrogated PTN-mediated CML colony formation (Figure $5 \mathrm{E}$ ).

In order to evaluate the effect of PTN on CML stem cell survival in a clinically relevant model, we treated splenic KSL cells from BA mice with imatinib (IM), a TKI utilized in the treatment of patients with CML, with and without PTN. IM treatment for 72 hours significantly increased the percentages of annexin ${ }^{+}$KSL cells, whereas the addition of $100 \mathrm{ng} / \mathrm{mL}$ PTN suppressed IMinduced CML cell death (Figure 5F). IM treatment also suppressed leukemic colony formation from KSL cells from BA mice, whereas the addition of PTN partially rescued leukemic colony formation from KSL cells (Figure 5G). Interestingly, IM treatment increased the expression of Ptn, Jun, and Atf4 in KSL cells from BA mice (Figure $5 \mathrm{H}$ ). In order to determine if PTN-mediated survival of CML cells was dependent on the function of c-Jun and the UPR, we treated KSL cells from BA mice with IM, with and without PTN, and with and without a c-Jun peptide inhibitor or the PERK inhibitor, GSK206414. PTN treatment suppressed IM-mediated CML stem cell apoptosis, and this effect was abolished by treatment with the c-Jun or PERK inhibitors (Figure 5I). These data suggest that PTN-mediated survival of CML stem cells is dependent on c-Jun and UPR activation.

Anti-PTN antibody suppresses human CML stem cell function. Because we observed that cell-autonomous PTN signaling was necessary for CML pathogenesis in mice, we sought to determine the role of PTN in regulating human CML growth. Newly diagnosed patients with chronic phase CML display significantly increased levels of PTN in the PB compared with healthy adults (Figure 6A). PTN expression is also increased by approximately 100-fold in $\mathrm{CD}_{3}{ }^{+}$cells from patients with chronic phase CML compared with $\mathrm{CD} 34^{+}$cells from healthy adults (Figure 6B). We also detected substantially increased concentrations of PTN in 72- hour cultures of primary human CML cells compared with cultures of BM cells from healthy adults, confirming that human CML cells secrete PTN at increased levels (Figure 6C). Expression of the gene encoding the PTN receptor, protein tyrosine phosphatase- $\zeta$ (PTP- $\zeta)$, was more than 10-fold increased in human CML CD $34^{+}$cells compared with $\mathrm{CD} 34^{+}$cells from healthy adults, whereas gene expression of another PTN receptor, anaplastic lymphoma kinase (ALK), was not increased in CML cells compared with healthy CD34+ cells (Figure 
6D). Flow cytometric analysis confirmed a significant increase in PTP- $\zeta$ protein on the surface of human CML CD $34^{+}$cells compared with healthy donor $\mathrm{CD} 34^{+}$cells (Figure $6 \mathrm{E}$ ). Surface expression of ALK protein was not different on $\mathrm{CD}^{+} 4^{+}$cells from patients with CML versus healthy donors (Supplemental Figure 8A).

In order to determine whether PTN inhibition could affect human CML growth, we plated primary human CML CD34 ${ }^{+}$cells in methylcellulose with and without $50 \mu \mathrm{g} / \mathrm{mL}$ anti-human PTN antibody and measured leukemic colony formation at day +14 (Figure 6F). Anti-human PTN antibody treatment significantly decreased human CML colony formation in vitro. In contrast, anti-human PTN antibody treatment had no inhibitory effect on colony formation from $\mathrm{CD} 34^{+}$cells from healthy adults, suggesting differential sensitivity of human CML cells to PTN inhibition (Figure 6F). Against 5 different human CML patient samples, anti-human PTN antibody treatment caused more than 50\% reduction in leukemic colony formation, with little inhibitory effect on $\mathrm{CD} 4^{+}$cells from healthy donors (Figure 6F). Anti-PTN antibody also suppressed CML colony formation of KSL cells from $\mathrm{BA} ; \mathrm{PTN}^{+/+}$mice and had no effect on CML colony formation of KSL cells from BA;PTN ${ }^{-/-}$mice, suggesting specificity of this antibody for PTN (Supplemental Figure 8B). Anti-PTN antibody also differentially inhibited colony growth from $\mathrm{PTN}^{+/} \mathrm{KSL}$ cells from BA mice, while displaying no inhibitory effect on $\mathrm{PTN}^{+/-} \mathrm{KSL}$ cells from NL C57BL/6 mice (Supplemental Figure 8B).

Treatment with anti-PTP- $\zeta$ antibody suppressed PTN-mediated expansion of colony-forming cells (CFCs) from KSL cells from BA mice and from NL KSL cells, whereas the addition of the specific ALK inhibitor, TAE684, did not significantly inhibit PTN-mediated CFC growth from KSL cells from BA mice or NL KSL cells (Supplemental Figure 8C). These data suggest that PTN regulates the growth of CML cells and NL HSPCs primarily through PTP- $\zeta$.

In order to determine if PTN inhibition could suppress human CML stem cell function, we cultured primary human CML CD34 ${ }^{+}$ cells with and without $50 \mu \mathrm{g} / \mathrm{mL}$ anti-human PTN antibody for 72 hours before i.v. injection into irradiated NOD/SCID IL-2receptor $\gamma$-null (NSG) mice (Supplemental Figure 8D). At 16 weeks after transplant, mice transplanted with $\mathrm{CML}$ cells treated with anti-human PTN antibody displayed significantly decreased engraftment compared with mice transplanted with the identical dose of human CML cells treated with IgG (Figure 6G). In a second human CML xenograft model, K562 cells were injected s.c. above the kidney capsule in adult NSG mice, and the mice were treated with $2 \mathrm{mg} / \mathrm{kg}$ anti-human PTN or isotype every Monday, Wednesday, and Friday. At 2 weeks after injection, CML tumors were measured in the NSG mice (Figure 6H). Mice treated with anti-human PTN antibody displayed significantly smaller tumor masses (Figure 6H). An additional group of mice was injected with K562 cells that were pretreated with $1 \mu \mathrm{M}$ IM for 72 hours before injection. IM pretreatment significantly decreased tumor size in vivo at 2 weeks after injection. Anti-human PTN further reduced tumor size compared with IM treatment alone (Figure 6H). These results suggest an additive effect of anti-human PTN antibody with TKI against human CML. Taken together, these results suggest that cell-autonomous secretion of PTN regulates human CML growth, and targeted inhibition of PTN can suppress human CML cell-repopulating capacity in vivo. Separately, we analyzed PTN expression in human AML data sets using the BloodSpot database $(43,44)$ and determined that PTN expression is also increased in human AML cells compared with NL human HSPCs (Supplemental Figure 8E). These data suggest high relevance for PTN expression in human myeloid leukemias.

\section{Discussion}

TKI therapy produces molecular remissions in more than $90 \%$ of patients with CML (45). Several studies have shown, however, that more than half of patients with CML who stop TKI treatment will have molecular recurrence within 2 years $(46,47)$. Underscoring this concern, patients with CML who experience cytogenetic and molecular remissions have been shown to have persistent populations of $\mathrm{BCR} / \mathrm{ABL}^{+} \mathrm{CD} 34^{+} \mathrm{CD} 38^{-}$stem cells despite up to 4 years of TKI therapy (48). These studies reinforce the necessity for longterm TKI therapy in most patients with CML. Additionally, a subset of CML stem cells are not dependent on BCR/ABL (49), and many BCR/ABL-expressing stem cells display resistance to TKI therapy (48-50). Up to $36 \%$ of patients taking IM were observed to develop BCR/ABL kinase mutations over time, representing an important basis for CML resistance to TKI therapy (51). Additional yet unknown mechanisms of CML stem cell resistance likely exist, and the successful discovery and targeting of such mechanisms could facilitate the eradication of CML stem cells in patients.

Recent studies have suggested an important role for BM microenvironment signals in regulating the development and progression of CML. CML stem cells depend upon CD44-mediated adhesion for homing and engraftment (18-20), and the BM microenvironment produces an altered cytokine milieu, which favors CML stem cell growth over NL HSCs (21). Furthermore, BM stromal cells promote CML stem cell resistance to TKIs via increased Wnt signaling and $\mathrm{N}$-cadherin interactions $(14,23)$. Targeted inhibition of nichederived signals, including IL-1 blockade and Wnt pathway inhibition, increases the elimination of CML in murine models $(14,22)$.

The contributions of autocrine mechanisms to CML pathogenesis are less well understood. Otsuka et al. reported that human CML cells did not demonstrate increased expression of hematopoietic cytokines compared with NL hematopoietic cells (27). Duncombe et al. showed that human CML cells secrete TNF and that TNF inhibits CML growth (25). Conversely, Gallipoli et al. reported that CML stem/progenitor cells secrete TNF- $\alpha$ and that TNF- $\alpha$ promotes CML cell survival (26). Autocrine expression of BMP4 has also been suggested to contribute to TKI resistance in human CML stem cells (52). Here, we demonstrate that murine and human CML stem cells upregulate and secrete PTN, a heparin-binding growth factor, that is not expressed substantially by NL HSPCs, but rather is produced in homeostasis by BM stromal cells and ECs (29). Further, we demonstrate that cellautonomous PTN signaling is necessary for CML stem cell growth in vitro, as well as the in vivo generation of phenotypic CML stem cells and CML disease initiation following transplantation. We recognize, however, that phenotype may not correlate with leukemia stem cell content and that single-cell transplantation studies would more accurately assess effects of PTN deletion on clonal CML stem cells compared with the bulk KSL cell transplantation studies performed here. Furthermore, PTN deletion may have affected the homing and proliferative potential of CML stem cells, as 
well as the leukemic microenvironment (53), in addition to effects on CML stem cell numbers. Human CML xenograft transplantation models are also limited because NSG mice may not recapitulate the human leukemic microenvironment. Nonetheless, our data suggest that PTN deletion abrogates CML disease initiation in transplanted mice, a hallmark of CML stem cell function. PTN expression also increased in murine CML stem cells treated with IM, suggesting that PTN expression may be a facultative survival response of CML stem cells to TKI. Ulbricht et al. reported that human astrocytomas express PTN and one of the PTN receptors, PTP- $\zeta$, and that PTN signaling regulated astrocytoma migration (54). Subsets of human multiple myeloma cells and $\mathrm{CD}^{+} 9^{+} \mathrm{B}$ acute lymphocytic leukemia cells also express PTN and anti-PTN antibody treatment suppresses the growth of myeloma cells and $\mathrm{B}$ acute lymphocytic leukemia cells in vitro $(55,56)$. PTN-mediated regulation of myeloid neoplasms has not been previously described. Here, we show that human $\mathrm{CML}$ CD $34^{+}$progenitors display a 100-fold increased expression of PTN compared with $\mathrm{CD}^{+}{ }^{+}$cells from healthy adults, a 10-fold increased expression of the PTN receptor (PTP- $\zeta)$, and anti-PTN treatment differentially suppresses human CML colony growth in vitro and engraftment in vivo. Consistent with our findings in human CML, PTN expression is also increased in datasets of human AML cells versus NL human HSPCs $(43,44,57)$. Taken together, these results suggest that PTN is a potentially important therapeutic target for human CML and AML.

We have previously shown that PTN promotes human HSC expansion in vitro and that PTN expression by BM stromal cells and ECs is necessary for HSC self-renewal and regeneration, respectively, in mice $(28,29)$. This finding raises an important question as to whether PTN can be therapeutically targeted in patients with CML without causing unintended hematopoietic toxicity. Our studies demonstrate that anti-PTN antibody suppressed human CML progenitor cell growth without affecting NL human CD34 ${ }^{+}$ cell growth in vitro. Further, over a range of PTN expression levels in KSL cells from homozygous $\mathrm{PTN}^{+/+}$and heterozygous $\mathrm{PTN}^{+/}$ mice, anti-PTN treatment differentially suppressed CML colony growth compared with that of NL hematopoietic progenitor cells. Mechanistically, PTN induces expression of the UPR genes, Xbp1 and Atf4, in CML stem cells, while not affecting the UPR machinery in NL HSPCs. Collectively, these results suggest that CML stem cells may have a relative addiction to PTN compared with NL HSPCs, and that a therapeutic window may exist for targeting PTN in patients with CML. Because CML stem cells that persist in patients are not BCR/ABL addicted (58), the combination of TKI with anti-PTN antibody represents a rational therapeutic strategy.

We observed that $\mathrm{PTN}^{+} \mathrm{CML}$ stem cells expressed significantly higher levels of Jun and the UPR genes, Xbp1 and Atf4, compared with PTN- deficient CML stem cells, and that PTN treatment induced the expression of Jun, Xbp1, and Atf4 in CML stem cells. Prior studies have shown that PTN can bind multiple different receptors, including $\mathrm{PTP}-\zeta$ and ALK, to promote the proliferation and survival of neurons $(59,60)$. CML stem cells from BA mice express increased levels of PTP- $\zeta$ compared with NL KSL cells, and PTP- $\zeta$ blockade suppressed PTN-mediated colony formation of CML stem cells, whereas inhibition of ALK had no effect. These results suggest that PTN-mediated growth of CML stem cells occurs primarily through PTP- $\zeta$. We also show that silencing of Jun, Atf4, or Xbp1 blocked PTN-mediated CML colony growth, and inhibition of c-Jun or PERK abrogated PTNmediated rescue of CML stem cells from TKI-induced apoptosis. These results suggest that PTN-mediated induction of CML stem cell growth and survival depends on activation of c-Jun and the UPR. Going forward, it will be important to determine the mechanism responsible for increased PTN expression in CML stem cells. Our data indicate that induction of Jun expression increases PTN expression in CML stem cells from BA mice. Because BCR/ABL induces the activation of $\mathrm{c}$-Jun, which is part of the activator protein 1 (AP1) transcription factor complex, and 2 AP1 binding sites have been identified in the $5^{\prime}$ promoter region of the PTN gene, we will prioritize evaluating the role of c-Jun in controlling PTN expression in CML stem cells $(40,41)$.

TKI therapy induces prolonged molecular and hematologic remissions in patients with CML, but cessation of TKI therapy results in molecular relapse in more than $50 \%$ of patients (46). Further, up to $20 \%$ of patients with CML fail TKI therapy, necessitating allogeneic stem cell transplantation (45). The inability to cure patients with CML with TKI therapy alone and the incidence of TKI treatment failures are indisputably related to the persistence of CML stem cells, which develop cell-autonomous and non-cell autonomous resistance mechanisms to BCR/ABL inhibition. We have identified a novel, cell-autonomous mechanism in which CML stem cells secrete PTN, which is essential for CML pathogenesis and for CML stem cell survival in vitro and in vivo. Targeted inhibition of PTN represents an attractive therapeutic strategy to eliminate CML stem cells as a complement to TKI therapy.

\section{Methods}

Mice. To generate the double-transgenic Scl/Tal1-tTA $\times$ BCR/ABL strain (2), SCL-tTA mice (strain no. 017722) and TRE-BCR/ABL mice (strain no. 006202) were obtained from the Jackson Laboratory. Doxycycline hyclate (Sigma-Aldrich) was administered in the drinking water at a concentration of $0.2 \mathrm{mg} / \mathrm{mL}$ to mating mice and newborn pups to suppress $B C R / A B L$ expression. At 4 weeks of age, doxycycline was withdrawn to induce $B C R / A B L$ expression in some mice. As previously described, embryos from mice bearing a constitutive deletion of Ptn (61) were obtained from the RIKEN Institute by the Jackson Laboratory and rederived in a C57BL/6 background. The Scl/Tal1-tTA and TRE-BCR/ABL mice were backcrossed 8 generations into the $\mathrm{PTN}^{-/-}$strain. As a result, all experimental mice described were in a C57BL/6 background. Sperm from PTN-GFP mice (Tg[Ptn-EGFP]HJ32Gsat MGI no. 4847351) developed as part of the GENSAT Project Rockefeller University was obtained from the MMRRC Repository and the strain was rederived in a C57BL/6 background. NSG mice (strain no. 005557) for human xenograft studies were purchased from the Jackson Laboratory.

Human patient samples. Newly diagnosed CML patient BM and $\mathrm{PB}$ samples were obtained from the UCLA Ronald Reagan Medical Center and the Fred Hutchinson Cancer Center (University of Washington) under a UCLA IRB-approved protocol (no. 15-000062). Deidentified, healthy control BM samples were obtained from AllCells. For PTN ELISA, PB serum samples from deidentifed patients were obtained from Discovery Life Sciences. 


\section{Table 1. Primer sequences for real-time PCR}

$\begin{array}{lll}\text { Gene } & \left.\text { Forward primer ( } \mathbf{5}^{\prime} \text { to } \mathbf{3}^{\prime}\right) & \text { Reverse primer }\left(\mathbf{5}^{\prime} \text { to } \mathbf{3}^{\prime}\right) \\ \text { CAPDH } & \text { AAGGTGAAGGTCGGAGTCAAC } & \text { GGGGTCATTGATGGCAACAATA } \\ \text { PTN } & \text { GGAGCTGAGTGCAAGCAAAC } & \text { CTCGCTTCAGACTTCCAGTTC } \\ \text { Ptprz1 } & \text { GCCTGGATTGGGCTAATGGAT } & \text { CAGTGCTCCTGTATAGGACCA } \\ \text { Alk } & \text { TCTCATCGCAGCCGATATGG } & \text { GGCATCTCCTTAGAACGCTCT }\end{array}$

Cell lines. The K562 cell line (ATCC, CCL-243) originally derived from the pleural effusion of a human patient with CML (42) was purchased from the ATCC and maintained in IMDM with 10\% FBS.

Histology. Reticulin staining of mouse femurs was performed by the UCLA Translational Pathology Core Laboratory on formalinfixed, decalcified femurs. For quantification, representative $\times 63$ fields of the marrow space without cortical or metaphyseal bone were captured from 5 distinct bones per treatment condition. The images were processed using Image (NIH) to determine the percentage of pixels in each image as follows. The color images were converted to 8-bit grayscale images. Reticulin-positive pixels were determined to have grayscale densities of 30 or less. This threshold was used to quantify the number of pixels positive for reticulin in each image.

Immunofluorescence imaging. Animals were injected via tail vein with $25 \mu \mathrm{g}$ of VE-Cadherin Alexa-647 antibody (Biolegend, 138006) 20 minutes prior to euthanasia. Spleens were dissected, rinsed, and placed in a cold $30 \%$ sucrose solution for 1 hour. The whole spleen was embedded in a cryomold with O.C.T. media. The cryomold was snap-frozen in a bath of dry ice $/ 100 \%$ ethanol. Longitudinal $5-\mu \mathrm{m}$ spleen sections were cut using a Leica Cryostat. Sections were fixed in $100 \%$ acetone for 5 minutes at $-20^{\circ} \mathrm{C}$. Sections were then rehydrated in PBS at room temperature for 20 minutes in the dark. Sections were stained overnight at $4^{\circ} \mathrm{C}$ with goat anti-mouse LepR antibody (R\&D Systems) in IHC staining buffer, rinsed 3 times with PBS, and then stained for 1 hour at room temperature with anti-goat AF594 (1:1000, R\&D Systems). After rinsing, slides were mounted in DAPI Pro-Gold mounting media.

Flow cytometry and annexin $V$ analysis. $\mathrm{PB}, \mathrm{BM}$, and spleens from mice were analyzed by flow cytometric analysis for myeloid, lymphoid, progenitor, and HSC subsets using the following antibodies purchased from BD Biosciences: B220 APC-CY7, Gr-1 PE, Mac-1 PE, lineage antibody cocktail V450, c-kit PE, sca-1 APC-Cy7, CD150 AF647, CD41 FITC, and CD48 FITC. A full list of antibodies utilized for flow cytometry is included in Supplemental Table 1. Discrimination of live and apoptotic cell fractions was performed by flow cytometry using the BD AnnexinV-FITC Apoptosis Detection Kit (BD Biosciences, 556570), following the manufacturer's instructions.

Isolation of splenic CD31+ ECs and LepR ${ }^{+}$cells. BM, spleen ECs, and LepR $\mathrm{R}^{+}$cells were isolated by FACS following methods previously described (29). Femurs and spleens were dissected, cleaned of adventitia, and crushed with a mortar and pestle. Crushed tissue was digested for 10 minutes at $37^{\circ} \mathrm{C}$ in a $1 \mathrm{mg} / \mathrm{mL}$ solution of Liberase (Sigma-Aldrich) in IMDM. Cells were rinsed in ice-cold PBS with 1\% FBS (Thermo Fisher Scientific) and depleted of lineage-positive cells with the Miltenyi lineage depletion kit (Miltenyi Biotec). For LepR $\mathrm{R}^{+}$cell isolation, the lineage-negative fraction was stained with
anti-CD45-V450, 7AAD, and LepR-PE to identify 7AAD-CD45-LepR ${ }^{+}$ cells. For EC isolation, the lineage-negative fraction was stained with anti-CD45-V450, 7AAD, and CD31-AF647. ECs were identified as the 7AAD ${ }^{-}$CD $45^{-}{ }^{-}$D $31^{+}$subset.

Murine CML repopulation assay. For the CML repopulation assays, $1 \times 10^{4}$ splenic KSL cells from $\mathrm{BA} ; \mathrm{PTN}^{+/+}$mice and $\mathrm{BA} ; \mathrm{PTN}^{-/-}$mice $\left(\mathrm{CD} 45.2^{+}\right)$mice were isolated. Recipient 10-week-old female CD45.1 ${ }^{+}$ B6.SJL mice (CD45.1 $1^{+}$) were irradiated with 950-cGy total body irradiation using a Cs137 irradiator. Donor KSL cells were delivered via tail vein injection along with a competing dose of $2 \times 10^{5}$ nonirradiated host BM cells. Multilineage donor hematopoietic cell engraftment was measured in the PB by flow cytometry, as previously described (62). The following antibodies purchased from BD Biosciences were used for this analysis: CD45.2 FITC, CD45.1 PE, B220 APC-CY7, Gr-1 PE, Mac-1 PE, CD3 V450, and Ter119 APC.

In order to isolate the deletion of PTN to the hematopoietic cell compartment, 10-week-old female CD45.1 $1^{+}$B6.SJL mice (CD45.1 $\left.1^{+}\right)$ were irradiated with 950-cGy total body irradiation and transplanted with $5 \times 10^{6} \mathrm{BM}$ cells from $\mathrm{BA} ; \mathrm{PTN}^{+/+}$mice $\left(\mathrm{CD} 45.2^{+}\right)$or $\mathrm{BA} ; \mathrm{PTN}^{-/-}$ mice. Both donor mice and recipients were maintained on doxycycline drinking water to suppress $B C R / A B L$ expression through 4 weeks after transplantation. After 4 weeks posttransplantation and verification of more than $90 \%$ donor CD $45.2^{+}$cell engraftment in the PB of all mice, doxycycline was withdrawn to allow BCR/ABL expression.

Survival studies and hematologic analysis of $B A$ mice. Comparative survival studies of $\mathrm{BA} ; \mathrm{PTN}^{+/+}$mice and $\mathrm{BA} ; \mathrm{PTN}^{-/-}$mice were repeated 4 times with 4 cohorts of 12 mice per genotype for a total of 48 mice per genotype. At 4 weeks of age, $B C R / A B L$ expression was induced in all mice by removal of doxycycline from the drinking water. Mice were monitored daily following doxycycline removal for signs of morbidity, and $\mathrm{PB}$ was sampled at 4-week intervals for complete blood counts. Per our UCLA Animal Research Committee-approved protocol (no. 2014-021-13L), mice were humanely euthanized if symptoms of morbidity such as extreme weight loss, immobility, or hunched posture were observed.

In vitro cultures of CML stem cells. The effect of cell-autonomous deletion of PTN from CML stem cells on in vitro expansion was measured by placing $10 \mathrm{KSL}$ cells from $\mathrm{BA} ; \mathrm{PTN}^{+/+}$mice and $\mathrm{BA} ; \mathrm{PTN}^{-/-}$mice in 96-well U-bottom plates. Cells were cultured in StemSpan medium supplemented with $125 \mathrm{ng} / \mathrm{mL}$ SCF and $20 \mathrm{ng} / \mathrm{mL}$ TPO. Cell counts were performed at days 3, 5, and 7. For assessment of receptor-ligand interactions in CML stem cells, $500 \mathrm{KSL}$ cells from C57BL/6 mice or KSL cells from BA mice were plated in 96-well U-bottom plates and cultured for 3 days in HSC-supportive media (IMDM supplemented with $10 \%$ FBS, $125 \mathrm{ng} / \mathrm{mL} \mathrm{SCF}, 50 \mathrm{ng} / \mathrm{mL}$ FLT-3 ligand, and $20 \mathrm{ng} / \mathrm{mL} \mathrm{TPO}$ ). Cells were treated with $200 \mathrm{ng} / \mathrm{mL}$ PTN (R\&D Systems) with and without $3 \mathrm{nM}$ ALK inhibitor TAE684 (Selleckchem) or $5 \mu \mathrm{g} / \mathrm{mL}$ anti-PTP- $\zeta$ (R\&D Systems). On day 3, cells were counted in each condition and then plated in 14-day CFC assays at 200 cells per 35-mm dish.

Leukemia gene expression analysis. The SA Biosciences Mouse Leukemia Pathway (PAMM-137Z, Qiagen) was used on an ABI Quant Studio 6-machine instrument to assay for gene expression differences in FACS-isolated splenic KSL cells from BA;PTN ${ }^{-/-}$mice and $\mathrm{BA} ; \mathrm{PTN}^{+/+}$ mice at 12 weeks after $B C R / A B L$ induction. Four biological replicates were used per group. The data were analyzed using the Qiagen GeneGlobe Data Analysis Center. Data were normalized using 5 housekeeping control genes: Actb, B2m, Gapdh, Gusb, and Hsp90ab1. Genes 
with CT values greater than 35 cycles were excluded. The $P$ values are calculated based on a Student's $t$ test of the replicate $2^{-\Delta C T}$ values for each gene in the control group and treatment groups.

Real-time PCR. RNA isolation was performed using Qiagen Micro RNeasy Kits. RNA concentrations were measured with the Nanodrop 2000 Spectrophotometer (Thermo Fisher Scientific). Five hundred nanograms of total RNA was reverse transcribed using the High Capacity cDNA Synthesis Kit (Thermo Fisher Scientific). Real-time PCR reactions were conducted using TaqMan Universal PCR Master Mix (Thermo Fisher Scientific) on an ABI Quant Studio 6-machine instrument with Taqman predesigned gene expression assays (Thermo Fisher Scientific). The following Taqman expression assays were used: mouse Atf4, Xbp1, Jun, LepR, and PTN, human PTN, human GAPDH, and mouse GAPDH. For detection of $B C R / A B L$ transcript levels, we used the primer sequences to detect the breakpoint of the BCR/ABL fusion gene (63). Forward primer: 5'-TCCACTCAGCCACTGGATTTAA-3'; reverse primer: 5'-GATGCTACTGGCCGCTGAA-3'; probe: 6-FAM-CAGAGTTCAAAAGCC-TGM. Primers and probes were produced by ABI and real-time PCR was performed on an ABI Quant Studio 6-machine instrument. For analysis of samples from patients with CML and healthy donors, we used validated primer sequences (64) to detect expression of GAPDH, PTN, Ptprz1, and Alk (Table 1).

Primers were purchased from Integrated DNA Technologies and real-time PCR reactions were performed using Sybr Green PCR Master Mix (Thermo Fisher Scientific) on an ABI Quant Studio 6-machine instrument. All PCR reactions were normalized to GAPDH levels and a reference sample using the ddCt method.

Induction and silencing of Jun expression. siRNA studies were performed in K562 cells using on-target plus siRNA constructs purchased from GE Dharmacon and Lipofectamine RNAiMAX. The siRNA transfection was conducted per the manufacturer's protocol for 48 hours in Opti-MEM reduced-serum media in a 24-well plate with 100,000 cells per well. The 10- $\mu \mathrm{M}$ siRNA stocks were diluted to a final concentration of $0.02 \mu \mathrm{M}$. After 48 hours, cells were collected and pelleted by centrifugation. Cells were then reseeded in 50,000 cells per well in 24-well plates with and without $500 \mathrm{ng} / \mathrm{mL}$ PTN for 3 days.

siRNA studies were performed using murine KSL cells and lin cells, and siRNA constructs were purchased from GE Dharmacon and Lipofectamine RNAiMAX. The siRNA transfection was conducted per the manufacturer's protocol for 60 hours in Opti-MEM reduced serum media in a 96-well plate with 20,000 cells per well. The 5- $\mu \mathrm{M}$ siRNA stocks were diluted to a final concentration of $50 \mathrm{nM}$. PTN was added to the designated conditions at $100 \mathrm{ng} / \mathrm{mL}$ for measurement of Atf4 and $X b p 1$ gene expression. After 60 hours, cells were collected and pelleted by centrifugation. Short-term induction of Jun expression was achieved via treatment with $10 \mu \mathrm{M}$ anisomycin added to serum-free media for 2 hours.

Chemical inhibitor assays. Fifty thousand K562 cells were cultured in IMDM plus $10 \%$ FBS with and without IM at $0.5 \mu \mathrm{M}$ or $1 \mu \mathrm{M}$ for 72 hours. Cells were treated with $40 \mu \mathrm{M}$ c-JUN peptide (Tocris). To inhibit PERK-mediated activation of Atf4, cells were treated with the PERK inhibitor, GSK2606414, used at $0.1 \mu \mathrm{M}$. GSK2606414 is a selective PERK inhibitor.

p-PERK analysis. Levels of p-PERK within BM KSL cells were measured as follows. BM cells from BA mice were isolated and stained live with lineage antibody cocktail V450, c-Kit-PE, and APC-Cy7 (Sca-1) to label the KSL population. Cells were then fixed and permeabilized using the BD Cytofix/Cytoperm Kit. Following permeabilization, cells were stained for 1 hour in permeabilization buffer with anti-PERK (phospho-T982, Abcam). Anti-PERK was preconjugated with AF488 using the Thermo Fisher Scientific AF488 Antibody Labeling kit. Following staining, cells were rinsed twice with permeabilization buffer prior to FACS analysis.

ELISA. Whole BM from 1 femur was flushed into $400 \mu \mathrm{L}$ PBS in a 1.5-mL Eppendorf tube. After centrifugation, BM supernatants were collected and analyzed for PTN protein levels using a mouse PTN ELISA kit (Lifespan Biosciences). For quantification of PTN levels in human PB serum, undiluted serum was assayed using the human PTN ELISA kit (Lifespan Biosciences).

Isolation of human cells. CD34 ${ }^{+}$cells were isolated from primary human $\mathrm{BM}$ aspirates and $\mathrm{PB}$ samples from newly diagnosed patients with CML as follows. Aspirates were diluted 1:1 in sterile PBS, carefully layered over GE Ficoll-Paque density gradient medium, and centrifuged at 1,400 rpm for 30 minutes. Mononuclear cells were collected and column enriched for $\mathrm{CD} 34^{+}$cells using the Miltenyi Biotec human $\mathrm{CD} 4^{+}$isolation kit.

CFC assays. CFC assays were performed as follows: 2,000 CD $34^{+}$ cells from patients with CML or healthy adults were seeded in 35-mm gridded CFC dishes in H4434 MethoCult media (Stem Cell Technologies). Anti-PTN or goat IgG (R\&D Systems) was added at a concentration of $50 \mu \mathrm{g} / \mathrm{mL}$ to the media. Colonies were scored at day 14 . For studies using the K562 cell line, each CFC dish was loaded with 500 cells. For evaluation of PTN inhibition in control versus CML stem cells, 2,000 KSL cells from C57BL/6 mice or BA mice were seeded in M3434 MethoCult media (Stem Cell Technologies). Anti-PTN or goat IgG (R\&D Systems) was added at a concentration of $50 \mu \mathrm{g} / \mathrm{mL}$ to the media. For assays of murine cells, each dish represents the progeny of a single well from a 96-well plate initiated with $2 \times 10^{3}$ to $8 \times 10^{3} \mathrm{KSL}$ cells and cultured for 72 hours.

Human CML transplantation model. Prior to transplantation, $1 \times 10^{5}$ human $\mathrm{CD}_{34}{ }^{+}$cells isolated from PB samples from newly diagnosed patients with CML were seeded in 96-well U-bottom cell culture plates. Cells were grown for 72 hours in StemSpan media (StemCell Technologies) supplemented with $1 \times$ Myeloid Expansion supplement (MES; contains SCF, TPO, G-CSF, and GM-CSF), $20 \mathrm{ng} / \mathrm{mL} \mathrm{IL-3,} 20 \mathrm{ng} / \mathrm{mL}$ IL-6, and $100 \mathrm{ng} / \mathrm{mL}$ fms-like tyrosine kinase 3. Additionally, wells contained either $50 \mu \mathrm{g} / \mathrm{mL}$ anti-human PTN (R\&D Systems, AF-252$\mathrm{PB}$ ) or goat IgG. After 72 hours, the cells were collected and counted. NSG mice conditioned with 300-cGy radiation were transplanted with the progeny of $4 \times 10^{5} \mathrm{CD} 34^{+}$cells per mouse. At 16 weeks after transplant, mice were euthanized, and human $\mathrm{CD} 45^{+}$cell engraftment was analyzed in the BM by flow cytometric analysis.

Subcutaneous human CML model. Human CML tumors were initiated by s.c. injection of either $2 \times 10^{6} \mathrm{~K} 562$ cells or the progeny $2 \times 10^{6} \mathrm{~K} 562$ cells treated with $1 \mu \mathrm{M}$ IM for 72 hours. Cells were injected into the left hind flanks of NSG mice in $200 \mu \mathrm{L}$ Matrigel, as previously described (65). Mice were then treated with $2 \mathrm{mg} / \mathrm{kg}$ anti-human PTN antibody (clone 3B10) (66), generated, and purified by the UCLA Therapeutic Antibody Platform, or IgG, i.p., every Monday, Wednesday, and Friday for 14 days. Control mice developed tumors larger than $20 \mathrm{~mm}$ (maximum allowable tumor size per UCLA Animal Research Committee) by 2 weeks. At 2 weeks, all animals were euthanized and tumor masses were measured. 
The anti-human PTN antibody was generated as follows: The heavy- and light-chain variable regions of an anti-PTN antibody, obtained from patent US7888485 (clone 3B10, sequence IDs 03 and 08) were synthesized (Genscript) (66). Resulting DNA was subcloned into linearized pcDNA3.4 protein expression vectors in the context of mouse IgG2a and kappa constant regions. Vectors were transiently transfected into ExpiCHO cells (Thermo Fisher Scientific) according to the manufacturer's instructions for 12 days. Antibody was purified from clarified supernatants by affinity chromatography on MabSelect SuRe columns (GE Life Sciences) following the product protocols. Eluted protein was characterized by SDS-PAGE reducing and nonreducing gels, quantified by UV absorption at $280 \mathrm{~nm}$, and aliquoted at $1 \mathrm{mg} / \mathrm{mL}$ in PBS.

PTN expression in human AML versus NL HSPCs. We utilized the BloodSpot database (www.bloodspot.eu) to evaluate PTN expression in NL human HSPCs (data set GSE42519) compared with human AML cells from The Cancer Genome Atlas (TCGA) $(43,44,57)$. The data set contains 4 different probe sets, namely 211737_x_at, 209466_x_at, 208408_at, and 209465_x_at. All data were analyzed using Prism 8 software (GraphPad Software, Inc.). An $F$ test was performed to evaluate homogeneity of variances, and we subsequently utilized an unpaired Student's $t$ test with Welch's correction to calculate $P$ values. $P<0.05$ was considered significant.

Statistics. Values are represented as means \pm SEM, unless stated otherwise. GraphPad Prism 6.0 was utilized for all statistical analyses. All data were checked for NL distribution and similar variance between groups. The choice of statistical test was based on the numbers of groups and variables. In the case of 1-way and 2-way ANOVA analyses, reported $P$ values were based on multiple test corrections. Sample size for in vitro studies was chosen based on observed effect sizes and standard errors from prior studies. For animal studies, a power test was used to determine the sample size needed to observe a 2-fold difference in mean between groups with 0.8 power using a 2 -sided
Student's $t$-test. All animal studies were performed using sex- and agematched animals, with WT littermates as controls. Animal studies were performed without blinding of the investigator, and no animals were excluded from the analysis. Statistical details of each experiment can be found in the figure legends, including the numbers of replicates and $P$ values from comparative analyses that were performed.

Study approval. All animal procedures were performed in accordance with Animal Use Protocol no. 2014-021-13M (Principal Investigator, John Chute) approved by UCLA Animal Research Committee. All human sample acquisition and analyses were performed in accordance with UCLA IRB 15-000062-CR-00004 (Principal Investigator, Joshua Sasine), approved by the UCLA IRB.

\section{Author contributions}

HAH and JPC conceived of and designed the research. HAH, MR, TF, YZ, LS, MK, CMT, AP, JK, and LZ conducted experiments and analyzed data. HS and JPS analyzed data and provided reagents. G. Sapparapu and PMB produced and provided reagents. G. Schiller provided reagents. HAH and JPC wrote the manuscript. MR, CMT, and G. Schiller edited the manuscript.

\section{Acknowledgments}

This work was supported by NHLBI grant HL-086998-06 (to JPC), NIAID grants AI-067769 (to JPC) and AI-107333 (to JPC), the California Institute for Regenerative Medicine Leadership Award LA1-08014 (to JPC), NIAID grant AI-138331 (to HAH), the Tower Cancer Research Foundation Career Development Grant (to MR), the UCLA Jonsson Comprehensive Cancer Center Seed Grant (to MR), and the Damon Runyon Fellowship Award (to CT).

Address correspondence to: John P. Chute MD, 545 OHRC Building, 617 Charles E. Young Drive South, UCLA, Los Angeles, California, USA. Phone: 310.206.4929; Email: jchute@mednet.ucla.edu.
1. Savona M, Talpaz M. Getting to the stem of chronic myeloid leukaemia. Nat Rev Cancer. 2008;8(5):341-350.

2. Reynaud D, et al. IL-6 controls leukemic multipotent progenitor cell fate and contributes to chronic myelogenous leukemia development. Cancer Cell. 2011;20(5):661-673.

3. Sloma I, Jiang X, Eaves AC, Eaves CJ. Insights into the stem cells of chronic myeloid leukemia. Leukemia. 2010;24(11):1823-1833.

4. Dameshek W. Some speculations on the myeloproliferative syndromes. Blood. 1951;6(4):372-375.

5. Huntly BJ, et al. MOZ-TIF2, but not BCR-ABL, confers properties of leukemic stem cells to committed murine hematopoietic progenitors. Cancer Cell. 2004;6(6):587-596.

6. Passegué E, Jamieson CH, Ailles LE, Weissman IL. Normal and leukemic hematopoiesis: are leukemias a stem cell disorder or a reacquisition of stem cell characteristics? Proc Natl Acad Sci U S A. 2003;100(suppl 1):11842-11849.

7. Schepers K, Campbell TB, Passegué E. Normal and leukemic stem cell niches: insights and therapeutic opportunities. Cell Stem Cell. 2015;16(3):254-267.

8. Holtz MS, Forman SJ, Bhatia R. Nonproliferating
$\mathrm{CML} \mathrm{CD} 34^{+}$progenitors are resistant to apoptosis induced by a wide range of proapoptotic stimuli. Leukemia. 2005;19(6):1034-1041.

9. Copland M, et al. Dasatinib (BMS-354825) targets an earlier progenitor population than imatinib in primary CML but does not eliminate the quiescent fraction. Blood. 2006;107(11):4532-4539.

10. Jiang $X$, et al. Chronic myeloid leukemia stem cells possess multiple unique features of resistance to BCR-ABL targeted therapies. Leukemia. 2007;21(5):926-935.

11. Konig H, et al. Enhanced BCR-ABL kinase inhibition does not result in increased inhibition of downstream signaling pathways or increased growth suppression in CML progenitors. Leukemia. 2008;22(4):748-755.

12. Jamieson $\mathrm{CH}$. Chronic myeloid leukemia stem cells. Hematology Am Soc Hematol Educ Program. 2008;(1):436-442.

13. Agarwal P, Bhatia R. Influence of bone marrow microenvironment on leukemic stem cells: breaking up an intimate relationship. Adv Cancer Res. 2015;127:227-252.

14. Agarwal P, et al. Enhanced targeting of CML stem and progenitor cells by inhibition of porcupine acyltransferase in combination with TKI. Blood.
2017;129(8):1008-1020.

15. Ågerstam H, et al. IL1RAP antibodies block IL-1-induced expansion of candidate CML stem cells and mediate cell killing in xenograft models. Blood. 2016;128(23):2683-2693.

16. Bhatia R, McGlave PB, Dewald GW, Blazar BR, Verfaillie CM. Abnormal function of the bone marrow microenvironment in chronic myelogenous leukemia: role of malignant stromal macrophages. Blood. 1995;85(12):3636-3645.

17. Bowers M, Zhang B, Ho Y, Agarwal P, Chen CC, Bhatia R. Osteoblast ablation reduces normal long-term hematopoietic stem cell self-renewal but accelerates leukemia development. Blood. 2015;125(17):2678-2688.

18. Krause DS, Lazarides K, von Andrian UH, Van Etten RA. Requirement for CD44 in homing and engraftment of BCR-ABL-expressing leukemic stem cells. Nat Med. 2006;12(10):1175-1180.

19. Krause DS, et al. Differential regulation of myeloid leukemias by the bone marrow microenvironment. Nat Med. 2013;19(11):1513-1517.

20. Krause DS, Lazarides K, Lewis JB, von Andrian UH, Van Etten RA. Selectins and their ligands are required for homing and engraftment of BCR$\mathrm{ABL1}^{+}$leukemic stem cells in the bone marrow 
niche. Blood. 2014;123(9):1361-1371.

21. Zhang B, et al. Altered microenvironmental regulation of leukemic and normal stem cells in chronic myelogenous leukemia. Cancer Cell. 2012;21(4):577-592.

22. Zhang B, et al. Inhibition of interleukin-1 signaling enhances elimination of tyrosine kinase inhibitor-treated CML stem cells. Blood. 2016;128(23):2671-2682.

23. Zhang B, et al. Microenvironmental protection of CML stem and progenitor cells from tyrosine kinase inhibitors through $\mathrm{N}$-cadherin and Wnt- $\beta$-catenin signaling. Blood. 2013;121(10):1824-1838.

24. Jamieson $\mathrm{CH}$, et al. Granulocyte-macrophage progenitors as candidate leukemic stem cells in blast-crisis CML. $N$ Engl J Med. 2004;351(7):657-667.

25. Duncombe AS, et al. Tumor necrosis factor mediates autocrine growth inhibition in a chronic leukemia. JImmunol. 1989;143(11):3828-3834.

26. Gallipoli P, et al. Autocrine TNF- $\alpha$ production supports CML stem and progenitor cell survival and enhances their proliferation. Blood. 2013;122(19):3335-3339.

27. Otsuka T, Eaves CJ, Humphries RK, Hogge DE, Eaves AC. Lack of evidence for abnormal autocrine or paracrine mechanisms underlying the uncontrolled proliferation of primitive chronic myeloid leukemia progenitor cells. Leukemia. 1991;5(10):861-868.

28. Himburg HA, et al. Pleiotrophin regulates the expansion and regeneration of hematopoietic stem cells. Nat Med. 2010;16(4):475-482.

29. Himburg HA, et al. Distinct bone marrow sources of pleiotrophin control hematopoietic stem cell maintenance and regeneration. Cell Stem Cell. 2018;23(3):370-381.e5.

30. Kvasnicka HM, et al. Bone marrow features improve prognostic efficiency in multivariate risk classification of chronic-phase $\mathrm{Ph}(1+)$ chronic myelogenous leukemia: a multicenter trial. JClin Oncol. 2001;19(12):2994-3009.

31. Landberg N, et al. CD36 defines primitive chronic myeloid leukemia cells less responsive to imatinib but vulnerable to antibody-based therapeutic targeting. Haematologica. 2018;103(3):447-455.

32. Cripe LD, et al. Role for $\mathrm{c}$-jun $\mathrm{N}$-terminal kinase in treatment-refractory acute myeloid leukemia (AML): signaling to multidrug-efflux and hyperproliferation. Leukemia. 2002;16(5):799-812.

33. Rangatia J, et al. Elevated c-Jun expression in acute myeloid leukemias inhibits C/EBPalpha DNA binding via leucine zipper domain interaction. Oncogene. 2003;22(30):4760-4764.

34. Zhou C, et al. JUN is a key transcriptional regulator of the unfolded protein response in acute myeloid leukemia. Leukemia. 2017;31(5):1196-1205.

35. Lu M, et al. Opposing unfolded-protein-response signals converge on death receptor 5 to control apoptosis. Science. 2014;345(6192):98-101.

36. Tabas I, Ron D. Integrating the mechanisms of apoptosis induced by endoplasmic reticulum stress. Nat Cell Biol. 2011;13(3):184-190.

37. Harding HP, et al. An integrated stress response regulates amino acid metabolism and resistance to oxidative stress. Mol Cell. 2003;11(3):619-633.

38. Woehlbier U, Hetz C. Modulating stress responses by the UPRosome: a matter of life and death. Trends Biochem Sci. 2011;36(6):329-337.

39. Edwards DR, Mahadevan LC. Protein synthesis inhibitors differentially superinduce $c$-fos and c-jun by three distinct mechanisms: lack of evidence for labile repressors. $Е M B O J$. 1992;11(7):2415-2424.

40. Li YS, et al. Characterization of the human pleiotrophin gene. Promoter region and chromosomal localization. J Biol Chem. 1992;267(36):26011-26016.

41. Raitano AB, Halpern JR, Hambuch TM, Sawyers CL. The Bcr-Abl leukemia oncogene activates Jun kinase and requires Jun for transformation. Proc Natl Acad Sci U S A. 1995;92(25):11746-11750.

42. Lozzio BB, Lozzio CB. Properties of the K562 cell line derived from a patient with chronic myeloid leukemia. Int J Cancer. 1977;19(1):136.

43. Rapin N, et al. Comparing cancer vs. normal gene expression profiles identifies new disease entities and common transcriptional programs in AML patients. Blood. 2014;123(6):894-904.

44. Bagger FO, Kinalis S, Rapin N. BloodSpot: a database of healthy and malignant haematopoiesis updated with purified and single cell mRNA sequencing profiles. Nucleic Acids Res. 2019;47(D1):D881-D885.

45. Hochhaus A, et al. Long-term outcomes of imatinib treatment for chronic myeloid leukemia. N Engl JMed. 2017;376(10):917-927.

46. Etienne G, et al. Long-term follow-up of the French Stop Imatinib (STIM1) study in patients with chronic myeloid leukemia. JClin Oncol. 2017;35(3):298-305.

47. Rea D, et al. Discontinuation of dasatinib or nilotinib in chronic myeloid leukemia: interim analysis of the STOP 2G-TKI study. Blood. 2017;129(7):846-854.

48. Chu S, et al. Persistence of leukemia stem cells in chronic myelogenous leukemia patients in prolonged remission with imatinib treatment. Blood. 2011;118(20):5565-5572.

49. Hamilton A, et al. Chronic myeloid leukemia stem cells are not dependent on Bcr-Abl kinase activity for their survival. Blood. 2012;119(6):1501-1510.

50. Corbin AS, et al. Human chronic myeloid leukemia stem cells are insensitive to imatinib despite inhibition of BCR-ABL activity. JClin Invest. 2011;121(1):396-409.

51. Jabbour E, et al. Allogeneic stem cell transplantation for patients with chronic myeloid leukemia and acute lymphocytic leukemia after Bcr-Abl kinase mutation-related imatinib failure. Blood. 2006;108(4):1421-1423.

52. Grockowiak E, et al. Immature CML cells implement a BMP autocrine loop to escape TKI treatment. Blood. 2017;130(26):2860-2871.

53. Perez-Pinera P, Berenson J, Deuel TF. Pleiotrophin, a multifunctional angiogenic factor: mechanisms and pathways in normal and pathological angiogenesis. Curr Opin Hematol. 2008;15(3):210-214.

54. Ulbricht $U$, et al. Expression and function of the receptor protein tyrosine phosphatase zeta and its ligand pleiotrophin in human astrocytomas. J Neuropathol Exp Neurol. 2003;62(12):1265-1275.

55. Chen $\mathrm{H}$, et al. Pleiotrophin is highly expressed by myeloma cells and promotes myeloma tumor growth. Blood. 2007;110(1):287-295.

56. Du CX, et al. Elevated expression of pleiotrophin in lymphocytic leukemia CD19+ B cells. APMIS. 2014;122(10):905-913.

57. Cancer Genome Atlas Research Network, et al. Genomic and epigenomic landscapes of adult de novo acute myeloid leukemia. $N$ Engl J Med. 2013;368(22):2059-2074.

58. Corbin AS, Agarwal A, Loriaux M, Cortes J, Deininger MW, Druker BJ. Human chronic myeloid leukemia stem cells are insensitive to imatinib despite inhibition of BCR-ABL activity. JClin Invest. 2011;121(1):396-409.

59. Perez-Pinera P, Chang Y, Deuel TF. Pleiotrophin, a multifunctional tumor promoter through induction of tumor angiogenesis, remodeling of the tumor microenvironment, and activation of stromal fibroblasts. Cell Cycle. 2007;6(23):2877-2883.

60. Stoica GE, et al. Identification of anaplastic lymphoma kinase as a receptor for the growth factor pleiotrophin. J Biol Chem. 2001;276(20):16772-16779.

61. Himburg HA, et al. Pleiotrophin regulates the retention and self-renewal of hematopoietic stem cells in the bone marrow vascular niche. Cell Rep. 2012;2(4):964-975.

62. Himburg HA, et al. Dickkopf-1 promotes hematopoietic regeneration via direct and niche-mediated mechanisms. Nat Med. 2017;23(1):91-99.

63. Foley SB, et al. Expression of BCR/ABL p210 from a knockin allele enhances bone marrow engraftment without inducing neoplasia. Cell Rep. 2013;5(1):51-60.

64. Shi Y, et al. Tumour-associated macrophages secrete pleiotrophin to promote PTPRZ1 signalling in glioblastoma stem cells for tumour growth. Nat Commun. 2017;8:15080.

65. Beider K, et al. Combination of imatinib with CXCR4 antagonist BKT140 overcomes the protective effect of stroma and targets CML in vitro and in vivo. Mol Cancer Ther. 2014;13(5):1155-1169.

66. Rosenfield SM, et al. Pleiotrophin (PTN) expression and function and in the mouse mammary gland and mammary epithelial cells. PLoS One. 2012;7(10):e47876. 